ARTICLE

https://doi.org/10.1038/s41467-020-14685-3

\title{
Salmonella Typhimurium biofilm disruption by a human antibody that binds a pan-amyloid epitope on curli
}

Sarah A. Tursi ${ }^{1}$, Rama Devudu Puligedda², Paul Szabo³, Lauren K. Nicastro', Amanda L. Miller', Connie Qiu (1) 1, Stefania Gallucci ${ }^{1}$, Norman R. Relkin ${ }^{3}$, Bettina A. Buttaro ${ }^{1}$, Scott K. Dessain ${ }^{2,4 凶}$ \& C,agla Tükel (1D ${ }^{1,4 凶}$

Bacterial biofilms, especially those associated with implanted medical devices, are difficult to eradicate. Curli amyloid fibers are important components of the biofilms formed by the Enterobacteriaceae family. Here, we show that a human monoclonal antibody with panamyloid-binding activity ( $\mathrm{mAb} 3 \mathrm{H} 3$ ) can disrupt biofilms formed by Salmonella enterica serovar Typhimurium in vitro and in vivo. The antibody disrupts the biofilm structure, enhancing biofilm eradication by antibiotics and immune cells. In mice, $3 \mathrm{H} 3$ injections allow antibioticmediated clearance of catheter-associated S. Typhimurium biofilms. Thus, monoclonal antibodies that bind a pan-amyloid epitope have potential to prevent or eradicate bacterial biofilms.

\footnotetext{
${ }^{1}$ Department of Microbiology and Immunology, Lewis Katz School of Medicine, Temple University, Philadelphia, PA, USA. ${ }^{2}$ Lankenau Institute for Medical Research, Wynnewood, PA, USA. ${ }^{3}$ Weill Cornell Medical Feil Family Brain \& Mind Research Institute, New York, NY, USA. ${ }^{4}$ These authors contributed

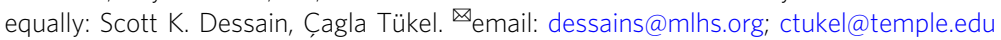


B iofilms are three-dimensional multicellular communities that allow bacteria to irreversibly adhere to indwelling medical devices and provide resistance to antibiotics and prevent eradication by innate immune cells. Biofilm-associated infections of indwelling medical devices are refractory to antibiotic treatment and require surgical debridement and/or device removal $^{1-7}$. Biofilm-producing enteric bacteria including Salmonella enterica and Escherichia coli remain to be the major cause of many bloodstream infections ${ }^{8-11}$. While biofilms of Salmonella play a critical role in persistent infections ${ }^{11}, E$. coli biofilms are important causes of prosthetic joint infections, recurrent urinary tract infections, and central line-associated blood infections (CLABSIs) $^{12-16}$.

Biofilms contribute to the development antibiotic tolerance and resistance ${ }^{17-19}$. Biofilms form a physical barrier to many antibiotics, restricting effective drug concentrations to sub-lethal levels and promoting the outgrowth of resistant strains ${ }^{20}$. They also block host immune responses, protecting cells from phagocytosis and complement mediated killing ${ }^{21-23}$. Lastly, biofilms enhance the ability of bacteria to generate "persister" sub-populations, which are multi-drug tolerant, essentially dormant cells with a characteristic gene expression signature ${ }^{24}$. Dispersal of a biofilm has the potential to seed a patient with drug-resistant bacteria $^{25,26}$. Strategies are needed to break down biofilms associated with indwelling medical devices and chronic infections, in order to render bacteria sensitive to antibiotics and susceptible to immune clearance.

Curli amyloid fibers are the major constituent (approximately $85 \%$ ) of the extracellular matrix in biofilms formed by the members of Enterobacteriaceae family, e.g. E. coli and Salmonella ssp. ${ }^{27-29}$. Curli is a heteropolymeric amyloid fibril comprising two subunits, Csga and CsgB, in a 20:1 ratio. CsgB nucleates CsgA fibril formation in the biofilm and attaches the amyloid fibrils to the bacterial surface ${ }^{30-34}$. Curli also promotes adhesion among bacteria within the biofilm and aids in surface attachment ${ }^{35,36}$. Amyloids such as curli form insoluble protein polymers that are characterized by a repeated cross-beta-sheet structure that can display conformational antibody-binding epitopes shared by amyloids of completely unrelated protein sequences ${ }^{37}$. Monoclonal antibodies (mAbs) that bind these conformational epitopes can inhibit polymerization of amyloidogenic proteins and disperse aggregated amyloids under some conditions.

$3 \mathrm{H} 3$ is a human mAb that preferentially binds amyloid beta protein $(A \beta)$ oligomers and fibrils, relative to $A \beta$ monomers, as well as many types of pathologic human amyloids, including immunoglobulin light chain and transthyretin amyloids ${ }^{38} .3 \mathrm{H} 3$ inhibits polymerization of $\mathrm{A} \beta$ and other amyloid precursors in vitro and reduces amyloid deposition in transgenic mouse models of AD and familial Danish dementia.

In this study, we investigate whether the $3 \mathrm{H} 3 \mathrm{mAb}$ has analogous effects against curli amyloids, using $S$. Typhimurium biofilms as an experimental model. We show that $3 \mathrm{H} 3$ inhibits the polymerization of curli, leading to alteration of the biofilm architecture and rendering the biofilm bacteria more sensitive to antibiotic treatment and to macrophage uptake. In addition, $3 \mathrm{H} 3$ inhibits biofilm formation on a vascular catheter in vivo and collaborates with an antibiotic to clear an established, catheterassociated biofilm.

\section{Results}

Anti-A $\beta$ mAb inhibits formation of $S$. Typhimurium biofilms. We tested the $3 \mathrm{H} 3 \mathrm{mAb}$ for activity against $S$. Typhimurium biofilm formation in vitro. We compared its activity to the $6 \mathrm{~A}$ isotype control $\operatorname{IgG}^{39}$ and three additional human mAbs, 4A6,
4G1, and 2C10, which were obtained from an Alzheimer's disease patient and preferentially bind to oligomeric $A \beta$ forms (Supplementary Table 1). The $3 \mathrm{H} 3$ has been previously observed to bind a pan-amyloid epitope. Of the new mAbs, only 4G1 showed potential pan-amyloid binding, as it and the $3 \mathrm{H} 3 \mathrm{mAb}$ both also bind to tau-paired helical filaments (PHF) (Supplementary Fig. 1). $4 \mathrm{G} 1$ also inhibits $A \beta$ fibril formation in vitro (Supplementary Fig. 2). As a negative control for biofilm formation, we used the isogenic $S$. Typhimurium mutant, $\operatorname{csg} B A$, which does not express curli ${ }^{40}$. We cultured $S$. Typhimurium under biofilminducing conditions on glass coverslips at $28^{\circ} \mathrm{C}$ for $72 \mathrm{~h}$ in the presence of $3 \mathrm{H} 3$, then rigorously washed the cultures and stained them with Syto9 green nucleic acid stain (bacteria) and Congo Red (CR) amyloid stain. We imaged the biofilms using confocal scanning laser microscopy.

Three-dimensional reconstructions of the z-stacks showed that the untreated $S$. Typhimurium biofilm was highly structured and covered most of the surface with an average thickness of $20 \mu \mathrm{m}$ (Fig. 1a), in comparison to the $\operatorname{csg} B A$ strain, which had no biofilm. The biofilm exposed to the control $6 \mathrm{~A}$ antibody was similar to the untreated control, $18.21 \mu \mathrm{m}$ thick and essentially confluent, although the depth of CR staining was reduced. Biofilms formed in cultures containing the other mAbs were substantially thinner, approaching the value for the $\operatorname{csg} B A$ mutant $(5.5 \mu \mathrm{m})$ : $4 \mathrm{~A} 6,10.9 \mu \mathrm{m} ; 4 \mathrm{G} 1,7.3 \mu \mathrm{m} ; 2 \mathrm{C} 10,9.5 \mu \mathrm{m}$; and $3 \mathrm{H} 3,7.5 \mu \mathrm{m}$ (Fig. 1b), with less surface coverage and CR staining (Fig. 1a). We quantitated the CR mean relative fluorescent unit (RFU) values for the biofilms. Three of the four mAb-exposed samples had significantly reduced CR staining: 4G1 (23.9 RFU), 2C10 (22.1 RFU), and $3 \mathrm{H} 3$ (23.2 RFU), compared to the control and 6A samples (47.1 RFU and 48.1 RFU, respectively), although these were greater than the $\operatorname{csgBA}$ biofilm (7.91 RFU) (Fig. 1c).

As the mechanism of biofilm formation may differ depending on available surfaces, we also tested the effects of the mAbs on pellicle formation (e.g. a biofilm at an air-liquid interface). $S$. Typhimurium strains were cultured in 96-well plates for 3 days under biofilm promoting conditions, in the presence or absence of the human mAbs, stained with crystal violet, and examined by visual inspection. Pellicle biomass formation was robust in the control and 6A mAb cultures (Fig. 1d). In contrast, the samples exposed to $3 \mathrm{H} 3$ had virtually no pellicle, similar to the isogenic $\operatorname{csg} B A$ mutant, and the pellicles developed with the $A \beta$-specific mAbs were thinner than the controls (Fig. 1d). To quantitate the extent of pellicle disruption, we repeated these experiments and measured the total crystal violet binding to washed and dried pellicles (Fig. 1d). The optical densities were significantly reduced for wells treated with all four anti-A $\beta$ mAbs. As $3 \mathrm{H} 3$ was the most effective of the mAbs evaluated, we investigated the affect of $3 \mathrm{H} 3$ on biofilm formation in several clinical isolates including S. enteritidis, S. pullorum, and uropathogenic E. coli UTI89 and E. coli MC4100, the common laboratory strain. Similar to our model organism, $S$. Typhimurium, $3 \mathrm{H} 3$ lead to a dose-dependent reduction in the pellicle biomass in S. enteritidis, E. coli UTI89, and E. coli MC4100 but not in S. pullorum (Supplementary Fig. 3). As the optical density for S. pullorum was overall lower compared to the other bacteria tested and $3 \mathrm{H} 3$ did not have an affect, it is possible that $S$. pullorum forms biofilms using bacterial factors than curli. Further experiments were conducted only with $3 \mathrm{H} 3$.

3H3 disrupts $S$. Typhimurium biofilm architecture and integrity. The biofilm formed in the presence of the $3 \mathrm{H} 3 \mathrm{mAb}$ showed a diffuse Syto9 staining pattern (Fig. 1a). We hypothesized that this represented a change in the density of the biofilm, such that bacteria within the biofilm would be more distant from the inert 

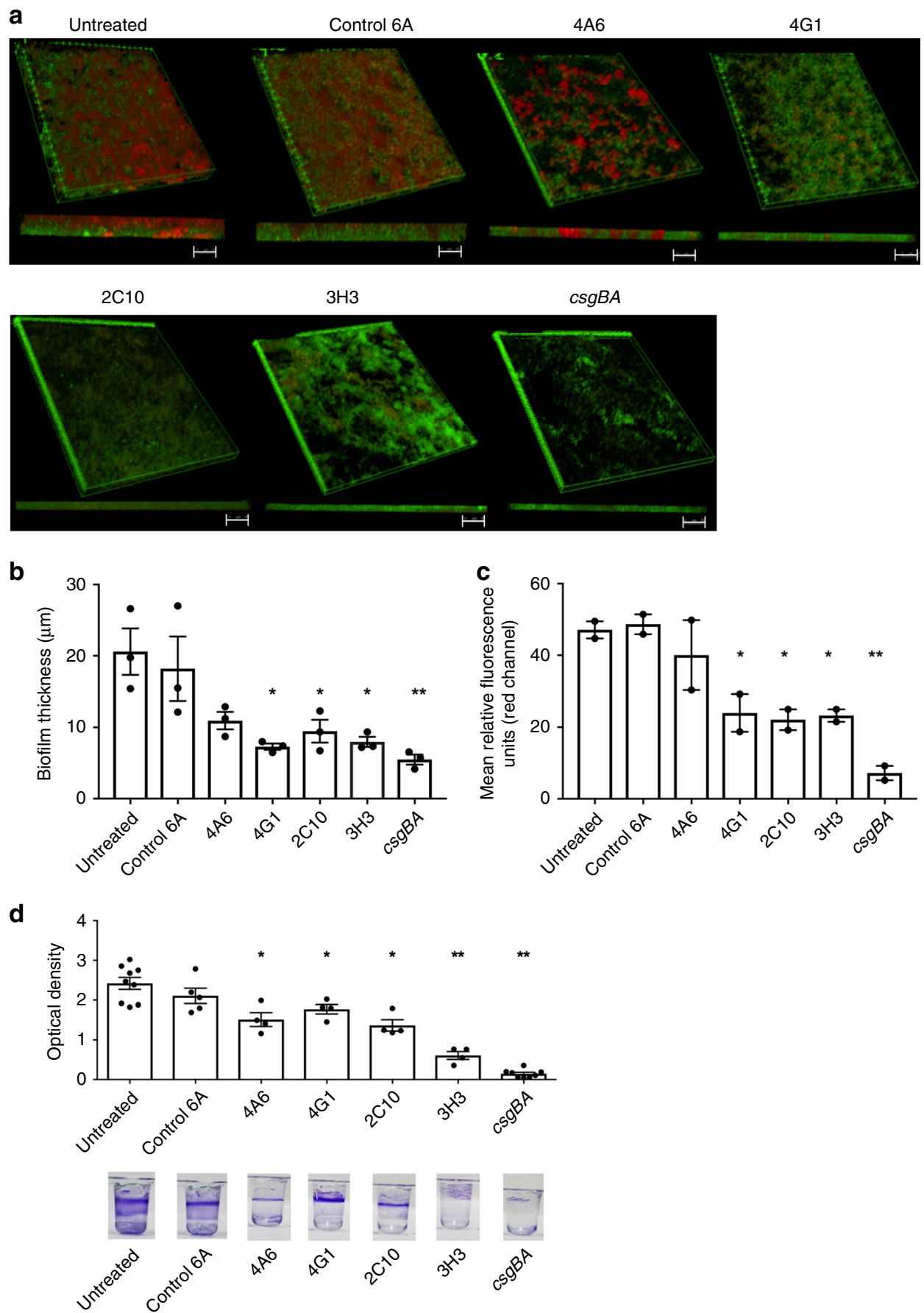

Fig. 1 Incubation of $\boldsymbol{S}$. Typhimurium biofilms with anti-amyloid mAbs reduces biofilm thickness and curli content. a $S$. Typhimurium was cultured in the absence of $\mathrm{mAb}$ (untreated) or in the presence of $0.5 \mathrm{mg} / \mathrm{ml}$ control antibody $6 \mathrm{~A}$ or $0.5 \mathrm{mg} / \mathrm{ml} 4 \mathrm{A6}, 4 \mathrm{G} 1,2 \mathrm{C} 10$, or $3 \mathrm{H} 3$. Isogenic mutant $\mathrm{S}$. Typhimurium $\operatorname{csgBA}$ was included as a negative control. After $72 \mathrm{~h}$, biofilms were stained with the bacterial stain Syto9 (green) and amyloid stain Congo Red (red), washed extensively, and imaged using a Leica TCS confocal microscopy at $\times 63$. ImageJ was used to create 3D reconstructions of z-stacks using the 3D projection application. Scale bars represent $25 \mu \mathrm{m}$. b Biofilm thickness $(\mu \mathrm{m})$ was determined from z-stacks using Leica TCS software. c Mean relative fluorescent units (RFU) of the red channel calculated from z-stacks using ImageJ. d Biofilms were grown in the absence (untreated) of antibody or in the presence of $0.5 \mathrm{mg} / \mathrm{ml} 6 \mathrm{~A}, 4 \mathrm{A6}, 4 \mathrm{G1}, 2 \mathrm{C10}$, or $3 \mathrm{H} 3$. csgBA was included as a negative control. After $72 \mathrm{~h}$, biofilms were stained with crystal violet, and the optical density at $570 \mathrm{~nm}$ was determined. Representative images of crystal violet staining are shown below the graph. Mean and SE were calculated from results from at least two independent experiments. ${ }^{\star} p<0.05,{ }^{\star \star} p<0.01$ as determined by Student's $t$-test.

surface and less tightly adherent. Fresh $S$. Typhimurium biofilms were prepared on glass coverslips for $72 \mathrm{~h}$, comparing cultures incubated with $6 \mathrm{~A}, 3 \mathrm{H} 3$, or a polyclonal anti-CsgA serum that is known to completely inhibit biofilm formation. In these experiments, the biofilms were very gently washed prior to imaging to prevent any possible disruption of the biofilms. Using ImageJ three-dimensional (3D) surface plot analysis, all particles within the biofilm were counted and pseudocolored green. This analysis revealed a difference in the topography of the biofilms (Fig. 2a). Curli-containing biofilms showed a dense topography of approximately $20 \mu \mathrm{m}$. However, the $3 \mathrm{H} 3$ and anti-CsgA exposed biofilms were less densely packed with an altered topography and 
a

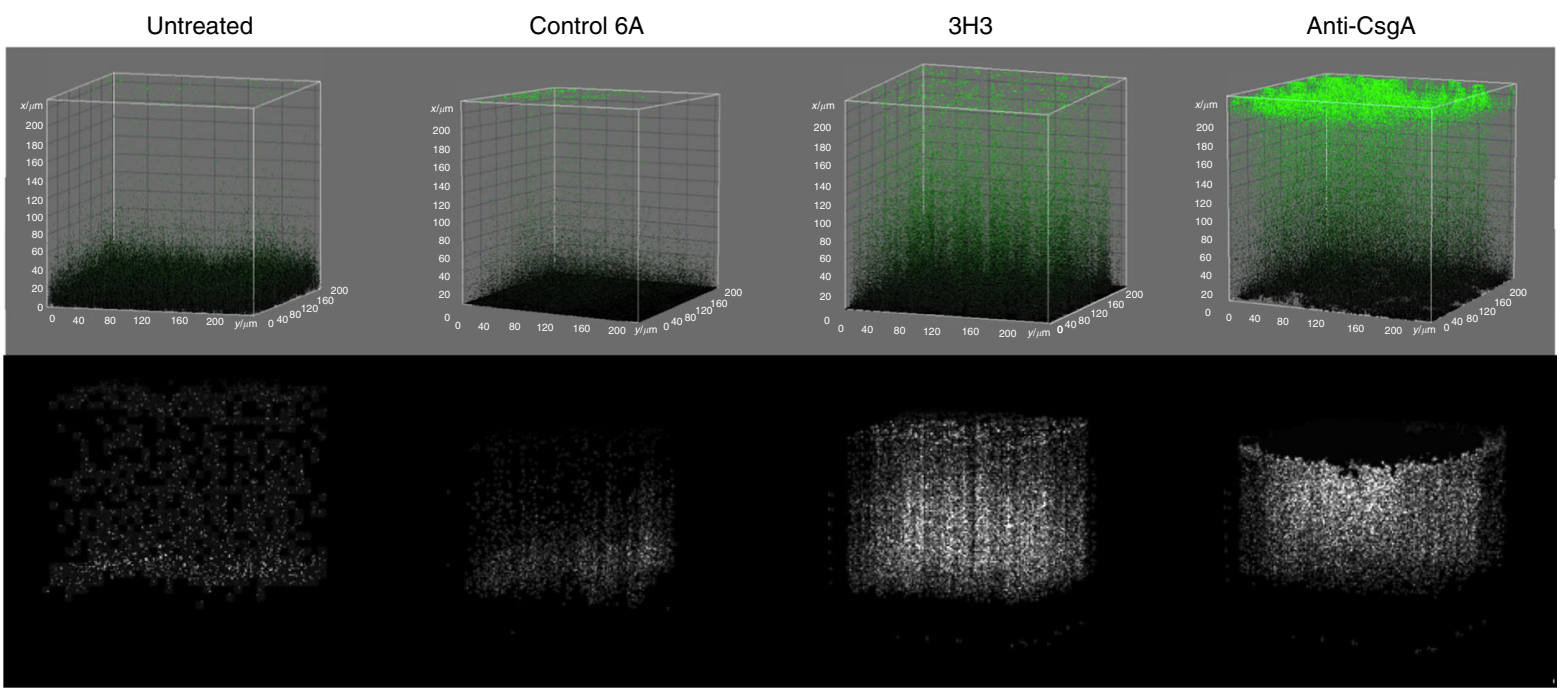

C

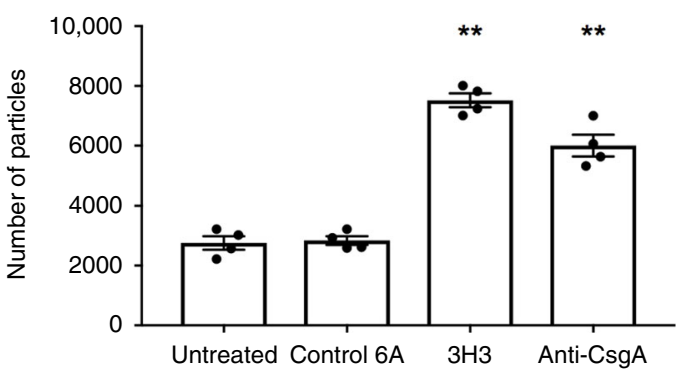

d

Untreated

$3 \mathrm{H} 3$ Anti-CsgA

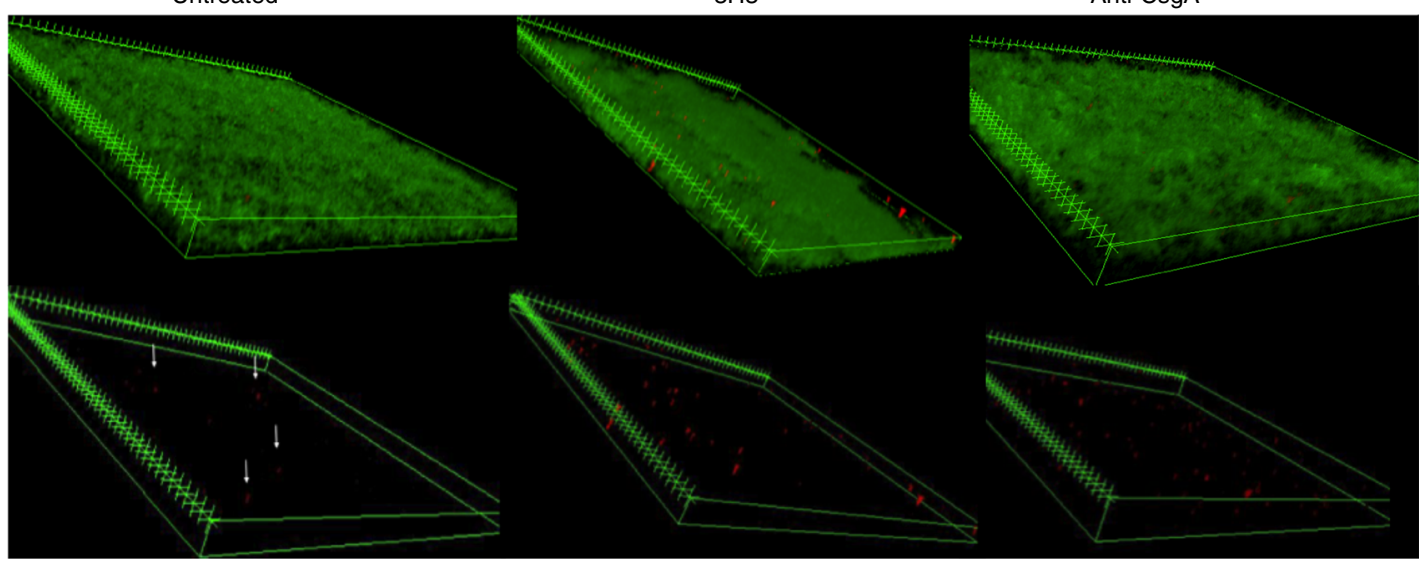

e

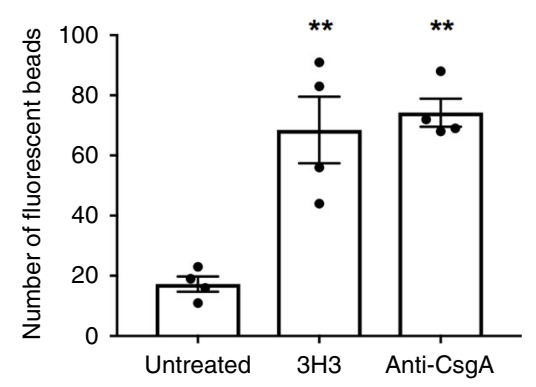

cells that appeared to be outside of the main biofilm mass (Fig. 2a). To quantify this population of cells, all particles above mean thickness of the untreated biofilm mass were identified and represented as white particles (Fig. 2b). The cells were counted using ImageJ (Fig. 2c). We next enumerated bacteria in the undisturbed supernatants by serial plating and found that biofilm disruption by $3 \mathrm{H} 3$ increased the release of bacteria (Supplementary Fig. 4).

These observations suggested the biofilm exposed to $3 \mathrm{H} 3$ may be more accessible to particles. We assessed the adherence and movement of $1 \mu \mathrm{m}$ red-fluorescent glyoxylate beads applied to the top of the biofilms. We tracked the movement of the beads over 
Fig. 2 Incubation with $\mathbf{3 H} \mathbf{3}$ alters the biofilm architecture. a S. Typhimurium biofilms were formed in the absence of mAb (untreated) or in the presence of $0.5 \mathrm{mg} / \mathrm{ml} 6 \mathrm{~A}, 0.5 \mathrm{mg} / \mathrm{ml} \mathrm{3H3}$, or anti-CsgA serum. After $72 \mathrm{~h}$, biofilms were stained with Syto9 (green) and visualized using Leica TCS confocal microscopy at $\times 63$. 3D surface plots were created in Image J and all Syto 9 particles of the biofilms appear as green. Particles are colored black at the bottom of the $z$-plane and are increased in green intensity from 0 to $220 \mu \mathrm{m}$ on the $z$-plane. $\mathbf{b}$ Particles above the mean biofilm mass of the untreated sample appear in white using the 3D surface plot application of ImageJ. c Number of particles above the mean biofilm mass (white particles) enumerated using ImageJ. d S. Typhimurium biofilms formed in the absence of mAb (untreated) or in the presence of $0.5 \mathrm{mg} / \mathrm{ml} 6 \mathrm{~A}, 0.5 \mathrm{mg} / \mathrm{ml} 3 \mathrm{H} 3$, or anti-CsgA. After $72 \mathrm{~h}$, biofilms were stained with Syto9 (green) and incubated with $10 \mu \mathrm{l}$ Crimson FluoSpheres $1 \mu \mathrm{m}$ red-fluorescent glyoxylate beads (red). Biofilms were imaged using confocal microscopy and biofilm projections of z-stacks were created using ImageJ (3D projections application). Scale bars represent $25 \mu \mathrm{m}$. Fluorescently labeled beads (red) were visualized by removing the Syto9 green channel. ImageJ was used to create 3D reconstructions of z-stacks. White arrows indicate location of beads in the untreated sample. e Number of red Crimson FluoSpheres within biofilms enumerated using ImageJ. Mean and SE were calculated from results of at least three independent experiments. ${ }^{\star} p<0.05,{ }^{\star \star} p<0.01$ as determined by Student's $t$-test.

20 min using time-lapse confocal microscopy (Fig. 2d, e, Supplementary Movies 1-3). In the control $S$. Typhimurium biofilm, vertical movement of the beads was observed, which was most likely caused by material properties of the biofilm. In biofilms formed with $3 \mathrm{H} 3$ or anti-CsgA, bead movement occurred both vertically and horizontally throughout the biofilm. Although equal amounts of beads were added to all samples, biofilms formed in the presence of $3 \mathrm{H} 3$ and anti-CsgA captured significantly more beads than the untreated controls. The average number of beads captured by the $S$. Typhimurium biofilms was 17 , whereas the $3 \mathrm{H} 3$ and anti-CsgA exposed biofilms captured 68 and 75 beads, respectively (Fig. 2f). Taken together, these data suggest that inhibition of amyloid deposition prevents formation of a mature biofilm matrix, resulting in loose bacterial association and a porous structure.

3H3 alters integrity of pre-established $S$. Typhimurium biofilms. To determine if $3 \mathrm{H} 3$ can alter integrity of pre-established biofilms, biofilms of $S$. Typhimurium were established for 24 or $48 \mathrm{~h}$. Biofilms were incubated for an additional $24 \mathrm{~h}$ in the presence or absence of $3 \mathrm{H} 3$ and then stained with Syto9, gently washed, and imaged using confocal microscopy. $3 \mathrm{H} 3$ exposure to biofilms established for 24 or $48 \mathrm{~h}$ inhibited further maturation of the biofilms and reduced total surface coverage (Fig. 3a). The $3 \mathrm{H} 3$ treated biofilms also displayed the characteristic unpacked topography (Fig. 3b). Visualizing the bacteria above the main biofilm mass as white particles allowed us to observe the incorporation of bacteria into the mature biofilm. $3 \mathrm{H} 3$ treatment reversed this process, even destabilizing the biofilm formed at the $48 \mathrm{~h}$ time point (Fig. $3 \mathrm{c}$ ), and increasing the number of bacteria dissociating from the biofilm (Fig. 3d).

3H3 inhibits the fibrillization of curli. We previously observed that $3 \mathrm{H} 3$ inhibits polymerization of amyloid fibrils from $\mathrm{A} \beta$ and a lambda immunoglobulin associated with primary amyloidosis ${ }^{38}$. To test whether $3 \mathrm{H} 3$ affects curli fibrillization, we tested the synthetic peptides $\mathrm{CsgA}_{\mathrm{R} 4-5}$ and $\mathrm{CsgAR}_{4-5 \mathrm{~N} 122 \mathrm{~A}}$ and the protein BSA in vitro using the Thioflavin $\mathrm{T}$ (ThT) assay ${ }^{41}$. Csg $\mathrm{A}_{\mathrm{R} 4-5}$, which has the sequence of the fourth and fifth repeats of CsgA, self-associates and forms fibrils, whereas $\operatorname{CsgAR}_{4-5 \mathrm{~N} 122 \mathrm{~A}}$ contains a single amino acid substitution that prevents fibrillization ${ }^{42}$. Incubation of $\mathrm{CsgA}_{\mathrm{R} 4-5}$ in the presence of $3 \mathrm{H} 3$ significantly reduced the polymerization of $\mathrm{CsgA}_{\mathrm{R} 4-5}$ alone or incubated with the control antibody 6A (Supplementary Fig. 5A). We also evaluated the lag time $\left(t_{0}\right)$ of fibrillization, the time required for monomers to self-associate and begin to fibrillize ${ }^{43}$. There was a significant increase in the lag time required for $\mathrm{CsgA}_{\mathrm{R} 4-5}$ to selfassociate when incubated with $3 \mathrm{H} 3$ in comparison to the calculated lag time for $\mathrm{CsgAR}_{4-5}$ alone. Overall these data suggest that $3 \mathrm{H} 3$ inhibits initiation of CsgA fibrillization as well as elongation of curli fibrils (Supplementary Fig. 5B).
$3 \mathrm{H} 3$ and antibiotic treatment synergizes for biofilm eradication. We reasoned that by altering the structure of the biofilm, $3 \mathrm{H} 3$ treatment would increase the susceptibility of the biofilm to antibiotics. We established biofilms of $S$. Typhimurium in the presence or absence of $3 \mathrm{H} 3$ for $48 \mathrm{~h}$ and then treated them with $30 \mu \mathrm{g} / \mathrm{ml}$ ampicillin for an additional $24 \mathrm{~h}$. Biofilms were then stained with Syto9, washed extensively, and visualized using confocal microscopy. No alteration in overall biofilm appearance was observed when biofilms were treated with ampicillin alone (Fig. 4a), and the average thickness of biofilms treated with ampicillin alone did not differ significantly from that of untreated biofilms (16.2 vs $14.8 \mu \mathrm{m})$ (Fig. $4 \mathrm{~b}) .3 \mathrm{H} 3$ treatment alone resulted in a biofilm with reduced surface coverage $(10.07 \mu \mathrm{m})$ compared to the untreated sample (Fig. 4a, b). Treatment with $3 \mathrm{H} 3$ and ampicillin together resulted in a biofilm with very little surface coverage (Fig. $4 \mathrm{a}$ ) and a biofilm thickness of $7.4 \mu \mathrm{m}$ (Fig. $4 \mathrm{~b}$ ). In addition to ampicillin, we also tested two additional classes of antibiotics ciprofloxacin and streptomycin, belonging to the fluoroquinolone and aminoglycoside drug classes, respectively, in combination with $3 \mathrm{H} 3$. Using the same method as described for ampicillin, we incubated $S$. Typhimurium biofilm with ciprofloxacin at $0.125 \mu \mathrm{g} / \mathrm{ml}$ or streptomycin $(12.5 \mu \mathrm{g} / \mathrm{ml})$ alone or in combination with $3 \mathrm{H} 3$ and visualized the biofilms using confocal microscopy. Both antibiotics were ineffective on the biofilm alone (Supplementary Fig. 6A, B). However, when S. Typhimurium biofilms were incubated with $3 \mathrm{H} 3$ and ciprofloxacin together for an additional $24 \mathrm{~h}$, there was a significant decrease in biofilm thickness and surface coverage in comparison to untreated or $3 \mathrm{H} 3$ treated biofilm (Supplemental Fig. 6A, B). Although not statistically significant, we observed a trend towards reduction in thickness and surface coverage in biofilms treated with $3 \mathrm{H} 3$ and streptomycin together (Supplemental Fig. 6A, B). Overall, these data show that $3 \mathrm{H} 3$-induced alterations to the biofilm extracellular matrix structure can facilitate antibiotic activity in vitro.

$3 \mathrm{H} 3$ promotes in vivo clearance of biofilms from pre-colonized catheters. Gram-negative bacteria are a common cause of CLABSIs $^{13,44}$. Biofilm-induced colonization of catheters leads to increased resistance to antibiotics and can necessitate catheter removal ${ }^{20}$. To investigate the effects of $3 \mathrm{H} 3$ on biofilm growth and architecture on catheters in vitro, we placed $1 \mathrm{~cm}$ sterile sections of an intravenous catheter in wells of a 24 -well plate that contained biofilm-inducing media. We added $3 \mathrm{H} 3$ or left the wells untreated under these conditions and inoculated the cultures with S. Typhimurium. After incubation at $28^{\circ} \mathrm{C}$ for $72 \mathrm{~h}$, biofilms were stained with Syto9 and Congo Red and visualized using confocal microscopy. Untreated biofilms grew within the lumen and adhered tightly to the walls of the catheters. Biofilms grown in the presence of $3 \mathrm{H} 3$ had a loose architecture and did not contain curli (Fig. 5a).

As host innate immune responses are an integral factor in controlling bacterial biofilms, we investigated whether $3 \mathrm{H} 3$ 

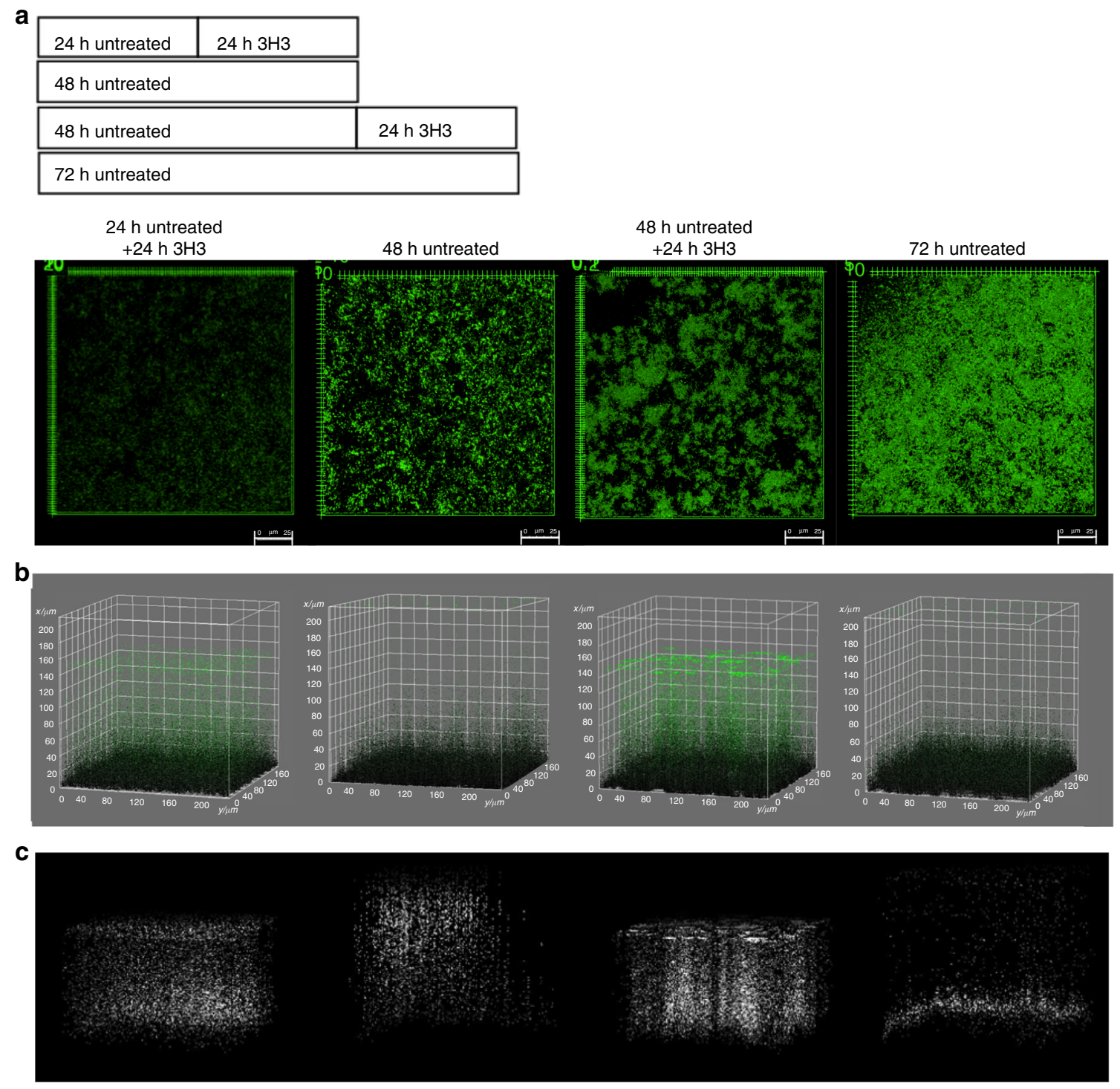

d

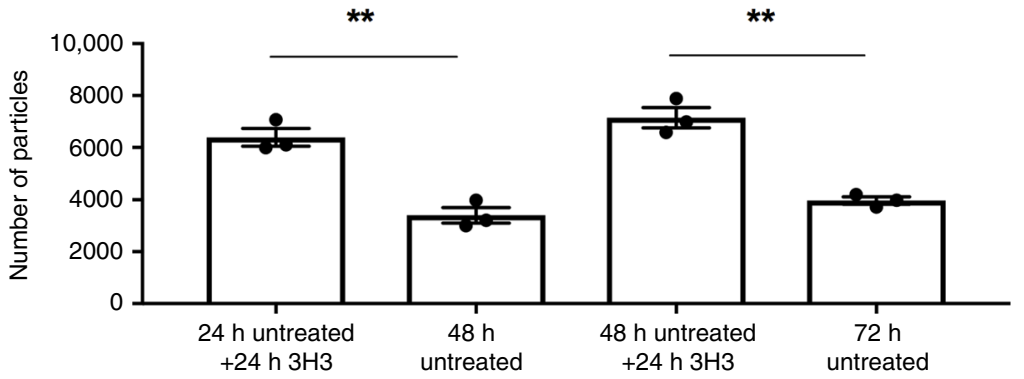

Fig. $3 \mathbf{3 H}$ alters the architecture of pre-established S. Typhimurium biofilms. a Experimental schematic and representative images of biofilms established for 24 and $48 \mathrm{~h}$ and then incubated for an additional $24 \mathrm{~h}$ without or with $0.5 \mathrm{mg} / \mathrm{ml} 3 \mathrm{H} 3$. Biofilms were stained with Syto9 (green) and visualized using Leica TCS confocal microscopy at $\times 63$. Scale bars represent $25 \mu \mathrm{m}$. b 3D surface plots of biofilms depicted in a created in ImageJ. All biofilm particles are colored green. c 3D surface plots in which particles above the mean biofilm mass appear in white as determined by the 3D surface plot application in ImageJ. d Number of particles above the mean biofilm mass enumerated using ImageJ. Mean and SE were calculated from results from at least three independent experiments. ${ }^{\star} p<0.05,{ }^{\star \star} p<0.01$ as determined by Student's $t$-test.

treatment of catheters pre-colonized with $S$. Typhimurium would enhance biofilm clearance in vivo. We pre-colonized intravascular catheters for $24 \mathrm{~h}$ with $S$. Typhimurium and then implanted the catheters subcutaneously into the back flanks of BALB/c mice. At 24 and $48 \mathrm{~h}$ after catheter insertion, $100 \mu \mathrm{g} 3 \mathrm{H} 3$ or buffer solution was percutaneously injected into the catheter lumens. At $72 \mathrm{~h}$ after catheter insertion, mice were euthanized and the catheters were removed. Excised catheters were stained with Syto9 and Congo Red and visualized using confocal microscopy. On catheters from cohorts of mice not treated with mAb, biofilms formed a dense matrix with bacteria encapsulated within curli (Fig. 5b, upper left). In contrast, catheters from cohorts that 
a

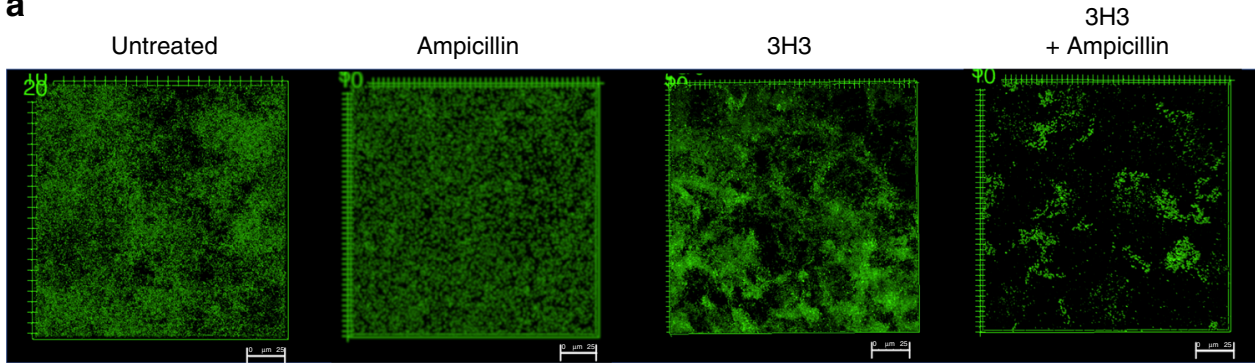

b

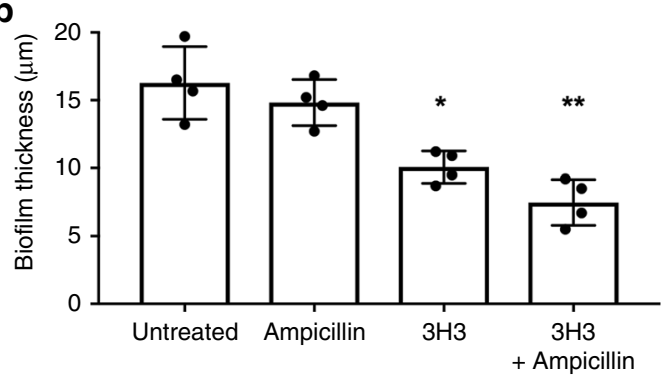

Fig. 4 Synergistic effect of $\mathbf{3 H} \mathbf{3}$ and antibiotic reduces $\mathbf{S}$. Typhimurium biofilm. a S. Typhimurium biofilms were untreated or were incubated in the presence of $0.5 \mathrm{mg} / \mathrm{ml}$ control antibody $6 \mathrm{~A}$ or $0.5 \mathrm{mg} / \mathrm{ml} 3 \mathrm{H3}$. After $48 \mathrm{~h}$, biofilms were subjected to $30 \mu \mathrm{g} / \mathrm{ml}$ ampicillin or not for an additional $24 \mathrm{~h}$. Biofilms were stained with Syto9 (green), washed extensively, and visualized using Leica TCS confocal microscopy at $\times 63$. Scale bars represent $25 \mu \mathrm{m}$. b Biofilm thickness $(\mu \mathrm{m})$ was determined from z-stacks using Leica TCS software. Mean and SE were calculated from results from at least three independent experiments. ${ }^{*} p<0.05,{ }^{* *} p<0.01$ as determined by Student's $t$-test.

received $3 \mathrm{H} 3$ had a loose biofilm architecture that was not tightly adhered to the catheters (Fig. 5b, upper right). To investigate the effect of a combination therapy of $3 \mathrm{H} 3$ with antibiotic on $S$. Typhimurium-colonized intravenous catheters in vivo, we treated mice with $1 \mathrm{mg} / \mathrm{ml}$ ampicillin in drinking water, starting $24 \mathrm{~h}$ prior to catheter insertion and continuing for the duration of the experiment. Catheters were injected percutaneously with 100 $\mu \mathrm{g} 3 \mathrm{H} 3$ or buffer solution 24 and $48 \mathrm{~h}$ after catheter insertion. At $72 \mathrm{~h}$, catheters were removed and stained as above. Ampicillin treatment alone did not impact the architecture of the $S$. Typhimurium biofilms (Fig. 5b, lower left). In contrast, catheters treated with ampicillin and $3 \mathrm{H} 3$ in combination were virtually free of biofilm, with only minimal levels of Congo Red staining (Fig. 5b, lower right).

In the in vivo experiment, we observed round, Congo Redpositive structures, about the size of phagocytic immune cells, on the catheters treated with $3 \mathrm{H} 3$ (Fig. 5b upper right) suggesting that immune cells had phagocytosed the biofilm-associated bacteria. We tested whether $3 \mathrm{H} 3$ can trigger phagocytosis of biofilmassociated bacteria by innate immune cells using bone marrowderived macrophages in vitro. We removed supernatants from untreated $S$. Typhimurium biofilms or from $S$. Typhimurium biofilms treated with $6 \mathrm{~A}, 3 \mathrm{H} 3$, or anti-CsgA serum, and applied them to macrophage monolayers. After $1 \mathrm{~h}$, we washed the macrophages to remove external bacteria and added gentamicin to kill any bacteria that had not been phagocytosed. Cells were then lysed to release the intracellular bacteria, and these bacteria were enumerated. Macrophages phagocytosed significantly more bacteria from the supernatants of the biofilm that had been incubated with $3 \mathrm{H} 3$ or anti-CsgA than from untreated supernatants or supernatants incubated with the $6 \mathrm{~A}$ control mAb (Fig. 6a). To account for possible differences in the numbers of bacteria in the supernatants, the supernatant optical densities were adjusted to 0.5 prior to the addition to macrophages. Again, more bacteria were recovered from the macrophages that were stimulated with the supernatants of the biofilm that had been incubated with $3 \mathrm{H} 3$ or anti-CsgA (Fig. 6b).
To investigate if $3 \mathrm{H} 3$ enhances the uptake of curli into immune cells, we added Congo Red-labeled curli fibers incubated with or without $3 \mathrm{H} 3$ for $1 \mathrm{~h}$ to a culture of immortalized macrophages. After $1 \mathrm{~h}$, cells were washed to remove excess curli. Curli internalization by the macrophages was visualized using confocal microscopy. More curli was detected inside the macrophages when it had been pre-incubated with $3 \mathrm{H} 3$ (Fig. 6c). These data suggest that $3 \mathrm{H} 3$ disrupts biofilm structure and enhances macrophage uptake of bacteria and curli fibers, which may promote clearance of biofilms by the innate immune system and reduce bacterial re-seeding.

\section{Discussion}

Curli fibrils are the major component of Enterobacteriaceae biofilms, which protect the bacteria from antibiotics and immune cells $^{28,35,45}$. Curli fibrils are amyloids, insoluble polymers that have a characteristic $\beta$-sheet structure ${ }^{30,46,47}$. Inhibiting curli polymerization has been proposed as a general model for treating Enterobacteriaceae biofilms ${ }^{48}$. Amyloid proteins with highly divergent sequences can share conformational epitopes that are recognizable by $\mathrm{mAbs}^{49,50}$. Some $\mathrm{mAbs}$ that bind pan-amyloid epitopes inhibit fibrillization of eukaryotic amyloids and have anti-amyloid activities in vivo ${ }^{38,49,51,52}$, but this has not been shown for prokaryotic amyloids.

Each of the four amyloid-binding mAbs tested here disrupted biofilm formation in vitro. $3 \mathrm{H} 3 \mathrm{had}$ been extensively characterized in previous studies ${ }^{38}$ and proved most effective. $3 \mathrm{H} 3$ is a human $\mathrm{mAb}$ specific for a pan-amyloid epitope that is present on a wide variety of pathogenic human amyloids, including $A \beta$, immunoglobulin light chain, transthyretin, and tau ${ }^{38}$. $3 \mathrm{H} 3$ inhibits amyloid fibril deposition in vitro and in animal models of $\mathrm{AD}$ and familial Danish dementia. Here, we describe how $3 \mathrm{H} 3$ also binds the bacterial amyloid curli and inhibits polymerization of curli monomers, which correlates with profound effects on the structure and function of the biofilm in vitro and in vivo. $3 \mathrm{H} 3$ $\mathrm{mAb}$ antagonized $S$. Typhimurium biofilm formation on glass 

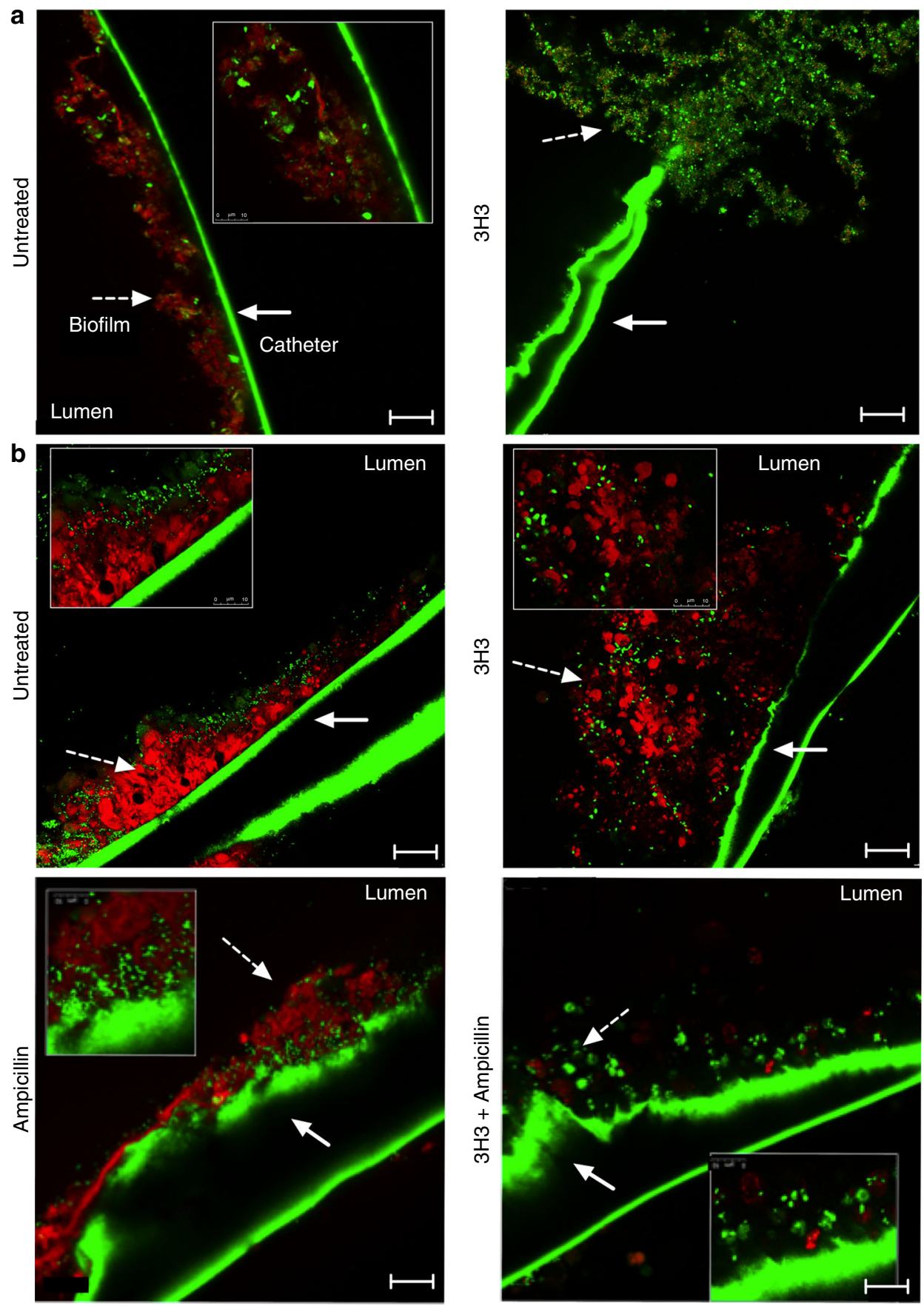

Fig. 5 Combination treatment of $\mathbf{3 H} \mathbf{3}$ and antibiotic leads to biofilm eradication. a Biofilms (dashed white arrow) were established in the presence (untreated) or absence of $0.5 \mathrm{mg} / \mathrm{ml} \mathrm{3H3}$ on medical grade catheters in vitro. After $72 \mathrm{~h}$ biofilms were stained with Syto9 (green) and amyloid curli stain Congo Red (red) and imaged using Leica TCS confocal microscopy at $\times 63$. Scale bars represent $25 \mu \mathrm{m}$. Inserts are $\times 3$ zoom images. Catheters exhibit green autofluorescence (solid white arrow). b Biofilms were established on medical grade catheters in vitro $24 \mathrm{~h}$ prior to insertion of catheters into flanks of mice. At 24 and $48 \mathrm{~h}$ post insertion, $100 \mu \mathrm{g} 3 \mathrm{H} 3$ was injected in vivo percutaneously into the catheter lumen. Where applicable, drinking water was supplemented with $1 \mathrm{mg} / \mathrm{ml}$ ampicillin beginning $24 \mathrm{~h}$ prior to catheter insertion. At $72 \mathrm{~h}$ after catheter insertion mice were euthanized and catheters were removed, stained with Syto9 (green) and Congo Red (red), and imaged using a Leica TCS confocal microscopy at $\times 63$. Scale bars represent $25 \mu \mathrm{m}$. Insets are $\times 3$ images. Catheters exhibit green autofluorescence.

slides, at the air-liquid interface (pellicles), and on vascular catheters. The extracellular matrix of the $3 \mathrm{H} 3$-treated biofilms failed to fully mature into an impermeable barrier with tightly embedded bacteria. Rather, we observed a "loose" topography, in which bacteria remained relatively distant from the surface and could be removed by washing. $3 \mathrm{H} 3$ also disrupted the structure of established $S$. Typhimurium biofilms. Treatment with $3 \mathrm{H} 3$ decompacted a 48 -h biofilm, allowing bacteria to move away from the surface. The decrease in density of the biofilm exposed to $3 \mathrm{H} 3$ was demonstrated by increased in penetration of $1 \mu \mathrm{m}$ glyoxylate beads in the biofilm. The $3 \mathrm{H} 3$-treated biofilm displayed changes in the extracellular matrix composition and structure, upon loss of curli incorporation which facilitated lateral movement of the glyoxylate beads within the biofilm. Finally, $3 \mathrm{H} 3$ enhanced the 

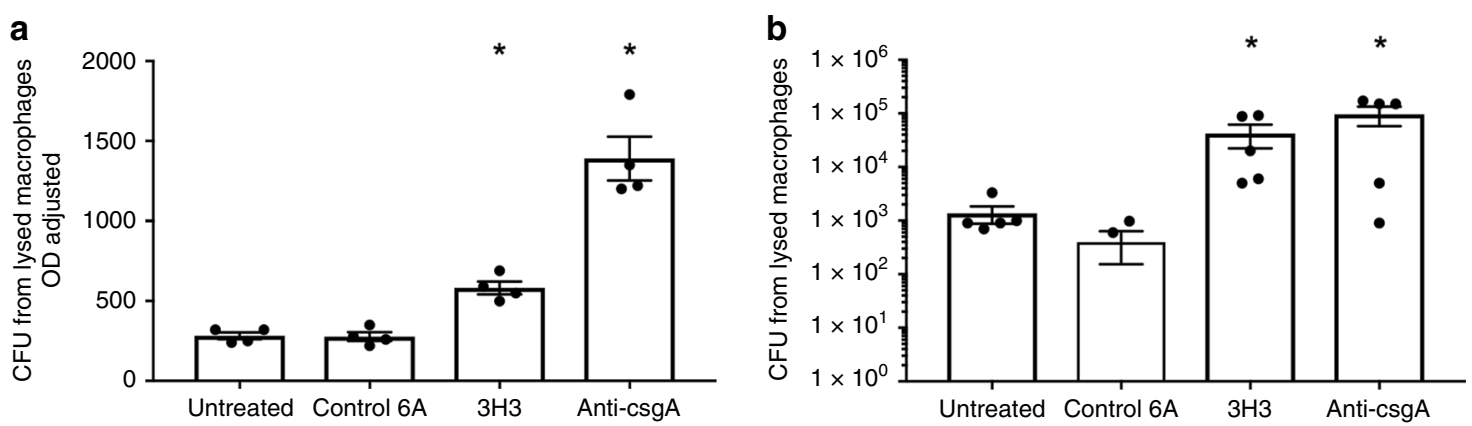

C
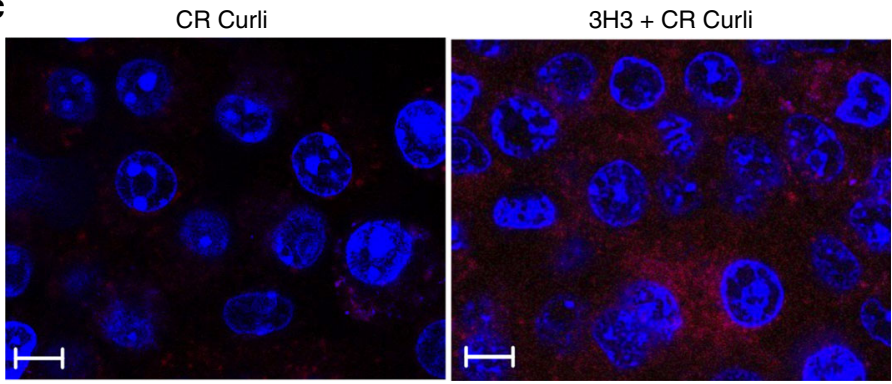

Fig. 6 Treatment with monoclonal antibody $3 \mathbf{H 3}$ enhances uptake of bacteria by macrophages. a Wild-type bone marrow derived macrophages (BMDMs) were treated with $100 \mu \mathrm{l}$ supernatants from biofilms grown in the absence (untreated) or presence of $0.5 \mathrm{mg} / \mathrm{ml} 3 \mathrm{H} 3$. After $1 \mathrm{~h}$, gentamicin was added, cells were lysed, and bacteria were enumerated as colony forming units. b Wild-type BMDMs were treated with supernatants from biofilms grown in the absence (untreated) or presence of $0.5 \mathrm{mg} / \mathrm{ml} 3 \mathrm{H} 3$ adjusted to an optical density of 0.5 at 600 . After $1 \mathrm{~h}$, gentamicin was added, cells were lysed, and bacteria were enumerated as colony forming units. c $1 \times 10^{6}$ wild-type immortalized macrophages were stimulated with $5 \mu \mathrm{g}$ of untreated curli (CR Curli) or $3 \mathrm{H} 3$ incubated curli (3H3 CR Curli) for $1 \mathrm{~h}$ at $37 \mathrm{C}$ and $5 \% \mathrm{CO}_{2}$. Cells were washed $3 \times$ with PBS to remove excess curli and macrophages were stained with DAPI (blue). Internalization of the curli (red) was imaged using a Leica TCS confocal microscopy at $\times 63$. Scale bars represent $25 \mu \mathrm{m}$. Mean and SE were calculated from results from at least three independent experiments. ${ }^{\star} p<0.05,{ }^{\star \star} p<0.01$ as determined by Student's $t$-test.

activity of ampicillin against biofilms formed on a glass slide and on a vascular catheter. Importantly, $3 \mathrm{H} 3$ collaborated with ampicillin to clear a biofilm from a vascular catheter implanted subcutaneously in mice. These data further support the concept of inhibiting curli polymerization as an anti-biofilm strategy and establish the therapeutic potential of the $3 \mathrm{H} 3 \mathrm{mAb}$ to treat curlicontaining biofilms.

Curli is secreted as a monomer (CsgA) that self-associates into small oligomeric subunits in the extracellular matrix. Small oligomeric subunits elongate though the addition of CsgA until the mature curli fibril is formed $28,31,36$. Curli amyloid shares structural and functional features with non-bacterial amyloids. Atomic resolution imaging revealed that curli amyloid fibrils resemble the $\mathrm{A} \beta$ fibrils found in Alzheimer's disease ${ }^{53}$, and synthetic D-enantiomeric peptides designed to inhibit $A \beta$ fibrillization also inhibit CsgA fibrillation. Curli fibrillization is also inhibited by human transthyretin, an amyloid protein responsible for cardiomyopathy and neuropathy ${ }^{54}$. These findings suggest the existence of panamyloid epitopes that are required for amyloid fibrillization and that polypeptides that bind these epitopes can be used to interrupt the maturation of amyloid structures.

Upon incubation of synthetic CsgA peptides with $3 \mathrm{H} 3$, we observed a reduction in curli fibrillization. $3 \mathrm{H} 3$ and $4 \mathrm{G} 1$ had the most activity against the $S$. Typhimurium biofilms, which correlated with relatively high binding affinity to $A \beta$ oligomers, in contrast to $4 \mathrm{~A} 6$ and $2 \mathrm{C} 10$, which had a strong preference for $\mathrm{A} \beta$ fibrils (Supplementary Table 1, Supplementary Fig. 2). In addition, $3 \mathrm{H} 3$ and $4 \mathrm{G} 1 \mathrm{mAbs}$ inhibited both curli and $\mathrm{A} \beta$ fibrillization, whereas 4A6 did not (Supplementary Fig. 2). The activity of $3 \mathrm{H} 3$ against curli is consistent with what we previously observed with $A \beta$ and suggests that the mAb binds a functional epitope on CsgA that is present on many human amyloids, even though its primary amino acid sequence differs greatly from the human proteins. Since the $3 \mathrm{H} 3$ was cloned from a healthy individual, it may be that the antibody originated as part of his immune response to a bacterial amyloid. Together, 3H3 and 4G1 define a category of mAbs that (a) bind pan-amyloid epitopes shared by eukaryotic and prokaryotic proteins and (b) inhibit amyloid fibrillization. Antibodies with these capabilities have utility for treating many different amyloid diseases.

Despite their functional similarities, $3 \mathrm{H} 3$ and $4 \mathrm{G} 1$ were cloned from different individuals and have highly divergent variable domain sequences with evidence of substantial affinity maturation (data not shown). These observations suggest that the curli amyloid polymerization domain contains multiple pan-amyloid epitopes that could be targeted by combinations of antibodies with complementary activities, potentially replicating the potent anti-fibrillar activity of the anti-CsgA antiserum. In addition, $3 \mathrm{H} 3$ and $4 \mathrm{G} 1$ variants (or new mAbs) may be discoverable that recognize CsgA-specific amino acids simultaneously with the pan-amyloid epitope. Such mAbs may be more potent antiamyloids because of higher affinity, increased steric hindrance, or allosteric effects. Lastly, it is possible that pan-amyloid-binding activity is not solely required for mAbs to inhibit curli polymerization. Instead, it may be that pan-amyloid epitopes define a particular region of curli important for oligomerization. An ideal $\mathrm{mAb}$ combination could target both pan-amyloid and curlispecific epitopes in this region.

The ability of $3 \mathrm{H} 3$ to inhibit polymerization of curli monomers suggests that some of its anti-biofilm activity may involve inhibiting the development of full-length curli fibrils. The observation that $3 \mathrm{H} 3$ binds and inhibits polymerization of amyloids with dramatically different primary sequences suggests that it binds a pre-fibrillar intermediate conformation that is shared by diverse 
amyloids. In the case of curli, these structures may consist of curli monomers or low molecular weight oligomers ${ }^{32}$. Maintenance of curli in an un-polymerized state could inhibit its incorporation into the extracellular matrix and would be consistent with the loose topography observed. However, $3 \mathrm{H} 3$ also demonstrated activity against established biofilms in vitro, loosening the biofilm topography and allowing bacteria to migrate away from the surface of the slide (Fig. 3). As curli is important for binding to abiotic surfaces $27,55,56$, it is possible that $3 \mathrm{H} 3$ interferes with the domains of curli required for surface binding.

Antibiotics that belong to the beta-lactam, fluoroquinolone, and aminoglycoside drug classes all demonstrated increased cell killing when the biofilm extracellular matrix was altered by $3 \mathrm{H} 3$. These experiments used previously determined MICs applicable to short-term exposures. However, the effects of biofilm disruption on antibiotic sensitivity will likely depend on whether the mechanism of cell killing is concentration dependent (fluoroquinolones, aminoglycosides) or time-dependent (beta-lactams). Additional studies will be necessary to explore these interactions in vitro and in vivo. Clearance of curli and curli-associated bacterial cells by phagocytic cells may have contributed to eradication of the vascular catheter biofilm in vivo. Antibodies have been shown to mediate macrophage-induced clearance of eukaryotic amyloids in vivo ${ }^{57-59}$. The biofilm exposed to $3 \mathrm{H} 3$ in vivo replicated the loose architecture seen in vitro but was also notable for Congo Red stained structures that were approximately the estimated size of phagocytic cells. Macrophage experiments showed that the 3H3-treated biofilms release bacteria in a form that can be phagocytosed by macrophages, and that uptake is enhanced by $3 \mathrm{H} 3$ binding to the bacteria/curli complexes.

A therapeutic strategy against biofilms needs to clear deviceassociated biofilms while reducing the development and spread of antibiotic resistance. For these purposes, a pan-amyloid $\mathrm{mAb}$ such as $3 \mathrm{H} 3$ offers many potential benefits. It could be given in combination with antibiotics to raise their effective concentrations sufficiently to clear infected medical devices. It could also be used as a device coating to prevent new biofilm formation. Bacteria should not develop resistance to pan-amyloid mAbs, as they would not affect the growth of planktonic bacteria. Furthermore, their ability to opsonize biofilm bacteria may reduce the systemic seeding that can occur when a biofilm is dissociated. Human $\mathrm{mAb}$ therapeutics have a strong safety record in general, and because the $3 \mathrm{H} 3$ was cloned from a healthy individual and binds a non-native antigen, it would be predicted to have few off-target effects. Human mAbs have half-lives of 2 weeks or more, which may be ideal for prevention and treatment of infections associated with curli biofilms. Lastly, as amyloids are widespread and found in biofilms of bacteria belonging to various phyla (Bacteroidetes, Proteobacteria, Firmicutes, and Thermodesulfobacteria) ${ }^{60,28}$ including important human pathogens such as Staphylococcus aureus $^{47}$, our findings may be generalizable to the treatment of many different bacterial biofilms.

\footnotetext{
Methods

Bacterial strains and growth conditions. S. Typhimurium strain IR715 is a fully virulent, nalidixic acid-resistant strain derived from the ATCC strain 14028 (ref. ${ }^{61}$ ). S. Typhimurium IR715 $c s g B A^{40}$ and $S$. Typhimurium IR715 $m s b B$ were previously described ${ }^{62}$. Bacteria were grown in Luria Bertani (LB) broth supplemented with $50 \mu \mathrm{g} / \mathrm{ml}$ nalidixic acid when appropriate. Clinical isolates of $S$. enteritidis and S. pullorum (ATCC 9120, isolated from a patient with diarrhea) were kindly provided by Dr. Andreas Baumler at UC Davis. Uropathogenic E. coli UTI89 (isolated from a patient with an acute bladder infection) and E. coli MC4100 were kindly provided by Dr. Scott Hultgren from Washington University in St. Louis. To induce biofilm formation, overnight bacterial cultures of Salmonella species were diluted 1:100 in LB No Salt medium and $E$. coli species were diluted 1:100 in LB low salt. All biofilm cultures were grown statically for $72 \mathrm{~h}$ at $28^{\circ} \mathrm{C}$. YESCA agar was prepared as described previously ${ }^{33}$
}

Isolation of antibodies. The $3 \mathrm{H} 3 \mathrm{mAb}$ has been previously described ${ }^{38}$. Additional $\mathrm{mAb}$ s reactive with oligomeric $\mathrm{A} \beta$ were cloned from $\mathrm{B}$ cells from an elderly female with a clinical diagnosis mild-moderate Alzheimer's disease (Supplementary Methods). The sample was collected under a protocol approved by the Main Line Hospitals Institutional Review Board and was consistent with the principles set out in the WMA Declaration of Helsinki and the NIH Belmont Report. The isotype control human mAb 6A (anti-botulinum toxin antibody) was described previously $^{39}$. Anti-CsgA serum was obtained from rabbits as previously described ${ }^{63}$.

Biofilm assays. The crystal violet assay was performed as described previously ${ }^{64}$ To investigate the curli content and thickness of biofilms grown in the presence or absence of antibody using confocal microscopy, overnight cultures of wild-type $S$. Typhimurium and the isogenic $\operatorname{csgBA}$ mutant were diluted 1:100 in LB No Salt broth with $0.5 \mathrm{mg} / \mathrm{ml}$ of $3 \mathrm{H} 3,4 \mathrm{G} 1,4 \mathrm{~A} 6,2 \mathrm{C} 10$, or anti-CsgA. Biofilm formation was analyzed at 24,48 , and $72 \mathrm{~h}$. Biofilms were washed four times to remove planktonic bacteria and then stained with $3 \mu \mathrm{l} / \mathrm{ml}$ Syto9 (Invitrogen) for $15 \mathrm{~min}$ and then washed four times with phosphate-buffered saline (PBS). Biofilms were then stained with $10 \mu \mathrm{g} / \mathrm{ml}$ Congo Red (Sigma Aldrich) for $15 \mathrm{~min}$ and washed four times with PBS. Congo Red was visualized at an excitation of $561 \mathrm{~nm}$ and an emission of $650-750 \mathrm{~nm}$ and Syto 9 was visualized at an excitation of $483 \mathrm{~nm}$ and an emission of $503 \mathrm{~nm}$ at $\times 63$ using a Leica SP5 Microscope equipped with a TCS confocal system. To image the overall architecture of the biofilms, the same protocol was used but the mature biofilms were only washed once with PBS and Congo Red was not applied. 3D surface plots were created using ImageJ Software

To investigate the increasing concentrations of $3 \mathrm{H} 3$ on biofilms bacteria were diluted 1:100 in LB No Salt broth with $0.1,1,10,25,50,250$, and $500 \mu \mathrm{g} / \mathrm{ml} 3 \mathrm{H} 3$ for $72 \mathrm{~h}$ statically at $28^{\circ} \mathrm{C}$. Crystal violet assay was performed.

Bacterial enumeration from biofilm supernatants. To enumerate bacteria present in supernatant due to altered biofilm architecture, $100 \mu \mathrm{l}$ supernatant was collected. Ten-fold serial dilutions were plated on LB agar plates with appropriate selection to determine the number of colony-forming units.

Culturing of macrophages. Immortalized macrophage cells (IMMs) derived from wild-type mice (NR-9456) were obtained from BEI Resources. IMMs were maintained in DMEM (Invitrogen) supplemented with 10\% heat-inactivated FBS (Life Technologies) and were grown in a humidified incubator at $37^{\circ} \mathrm{C}$ with $5 \% \mathrm{CO}_{2}$.

Bone marrow-derived macrophages (BMDMs) were generated from 6- to 8-weekold female wild-type C57BL/6 mice (Jackson Laboratories). Macrophages were isolated and differentiated as described previously ${ }^{65}$. The differentiated BMDMs were plated at $5 \times 10^{5}$ cells per well in a 48 -well plate and allowed to adhere for $24 \mathrm{~h}$ in a humidified incubator $\left(37^{\circ} \mathrm{C}\right.$ in $\left.5 \% \mathrm{CO}_{2}\right)$ prior to experimentation.

Purification of curli amyloid and Congo Red labeling. The purification of amyloid curli has been previously described ${ }^{66}$. The concentration of the curli fibers was determined using BCA reagent (Novagen) according to the manufacturer's protocol. Purified curli was labeled with Congo Red as described previously ${ }^{67}$.

Macrophage uptake assays. Overnight cultures of $S$. Typhimurium were diluted 1:100 in LB No Salt broth in the presence or absence of $0.5 \mathrm{mg} / \mathrm{ml} \mathrm{mAb}$ and grown statically at $28^{\circ} \mathrm{C}$. After $72 \mathrm{~h}, 100 \mu \mathrm{l}$ culture supernatant was collected and stained with $3 \mu \mathrm{l} / \mathrm{ml}$ Syto9. An additional aliquot of $100 \mu \mathrm{l}$ was added to BMDM monolayers in 24-well plates. Macrophages and bacteria were incubated in a humidified chamber at $37^{\circ} \mathrm{C}$ in $5 \% \mathrm{CO}_{2}$. After $1 \mathrm{~h}, 10 \mu \mathrm{g} / \mathrm{ml}$ gentamicin (Fisher) was added, and samples were incubated for $1 \mathrm{~h}$. BMDMs were washed three times with PBS and then lysed with $0.01 \%$ Triton X-100 for $30 \mathrm{~min}$ in a humidified chamber at $37^{\circ} \mathrm{C}$ in $5 \% \mathrm{CO}_{2}$. To enumerate the bacteria within the BMDMs, 1:10 serial dilutions of the BMDM lysate were collected, and the numbers of colony-forming units were determined by plating on agar plates. To visualize bacteria uptake, the BMDMs were first stained with $15 \mu \mathrm{M}$ CellTracker Blue (Molecular Probes) for $15 \mathrm{~min}$ according to the manufacturer's protocol and processed as above. Bacteria were stained with Syto9 and analyzed as described above.

To determine curli uptake, Congo Red-labeled curli $(100 \mu \mathrm{g})$ was incubated with or without $0.5 \mathrm{mg} / \mathrm{ml} 3 \mathrm{H} 3$ at room temperature with gentle shaking. After $1 \mathrm{~h}$, $5 \times 10^{5}$ wild-type IMMs were stimulated with $10 \mu \mathrm{l} / \mathrm{ml}$ Congo Red-labeled curli with or without $3 \mathrm{H} 3$. After $1 \mathrm{~h}$ at $37^{\circ} \mathrm{C}$ with $5 \% \mathrm{CO}_{2}$, cells were washed three times with sterile PBS and then incubated with $0.01 \%$ Triton X-100 for $30 \mathrm{~min}$ at $37^{\circ} \mathrm{C}$ in $5 \% \mathrm{CO}_{2}$. Cells were transferred to wells of a clear bottom, black, 96-well microplate. Fluorescence of Congo Red-labeled curli was measured using a Flex Station (Molecular Devices) at an excitation of $497 \mathrm{~nm}$ and emission at $614 \mathrm{~nm}$ using a top read.

To visualize the uptake of Congo Red-labeled curli by IMMs using confocal microscopy, Congo Red-labeled curli was incubated with or without $3 \mathrm{H} 3$. One day prior to stimulation, sterile circular glass coverslips (Fisher) were inserted into wells of sterile 24 -well tissue culture dishes, and each well was seeded with $1 \times 10^{6}$ wildtype IMMs. After the macrophages had adhered to the glass coverslip, cells were treated with $5 \mu \mathrm{g} / \mathrm{ml}$ Congo Red-labeled curli previously incubated with or without $0.5 \mathrm{mg} / \mathrm{ml} 3 \mathrm{H} 3$ and $1 \mu \mathrm{g} / \mathrm{ml}$ DAPI (Invitrogen). After $30 \mathrm{~min}$, cells were washed 
three times with sterile PBS. Coverslips were placed on slides with $3 \mu \mathrm{l}$ Vectashield $(\mathrm{H}-100)$. Cells were imaged using confocal microscopy at $\times 63$. Congo Red was visualized at an excitation of $497 \mathrm{~nm}$ and emission at $614 \mathrm{~nm}$, and DAPI was visualized at an excitation of $358 \mathrm{~nm}$ and an emission of $461 \mathrm{~nm}$.

Evaluation of biofilm topology with a bead assay. Pellicle biofilms of $S$. Typhimurium were cultured on glass coverslips and stained with Syto 9 as described above. After biofilms established, $10 \mu \mathrm{l}$ fluorescently labeled beads (Crimson FluoSpheres, $1.0 \mu \mathrm{m}$, Life Technologies) were added on top of the biofilm. After a 10-min incubation at room temperature, excess beads were removed by washing the biofilm gently with sterile PBS three times. Coverslips were removed from the dishes and placed upside down in wells of an eight-well Multi-test slide (MP Biomedicals) with $3 \mu \mathrm{l}$ Vectashield (H-1000) and sealed with fast drying clear nail polish (Fisher). Time-lapse bead movement was visualized using a Leica SP5 Microscope with a TCS confocal system taken at $\times 63$. Syto9 was visualized at an excitation of $483 \mathrm{~nm}$ and an emission of $503 \mathrm{~nm}$. Crimson FluoSpheres were visualized at an excitation of $625 \mathrm{~nm}$ and an emission of $645 \mathrm{~nm}$.

Antibiotic and $\mathbf{m A b}$ combination treatment of biofilms. To determine the effect of a combination of $\mathrm{mAb}$ and antibiotic treatment on biofilm formation, biofilm growth was induced in the presence or absence of $0.5 \mathrm{mg} / \mathrm{ml} 3 \mathrm{H} 3$, control $6 \mathrm{~A}$, or anti-CsgA. After $48 \mathrm{~h}, 30 \mu \mathrm{g} / \mathrm{ml}$ ampicillin was added. After an additional $24 \mathrm{~h}$, biofilms were washed with PBS and stained with Syto9 and imaged using confocal microscopy as described above. Overnight cultures of wild-type $S$. Typhimurium were diluted 1:100 in LB No Salt broth with or without $0.5 \mathrm{mg} / \mathrm{ml}$ of $3 \mathrm{H} 3$ and grown for $72 \mathrm{~h}$ at $28^{\circ} \mathrm{C}$. After $72 \mathrm{~h}, 0.125 \mu \mathrm{g} / \mathrm{ml}$ ciprofloxacin or $12.5 \mu \mathrm{g} / \mathrm{ml}$ streptomycin was added to appropriate biofilms for an additional $24 \mathrm{~h}$. Biofilms were washed four times to remove planktonic bacteria and then stained with $3 \mu \mathrm{l} / \mathrm{ml}$ Syto9 (Invitrogen) for $15 \mathrm{~min}$ and then washed four times with PBS. Syto9 was visualized at an excitation of $483 \mathrm{~nm}$ and emission of $503 \mathrm{~nm}$ at $\times 63$ using a Leica SP5 Microscope equipped with a TCS confocal system. 3D surface plots were created using ImageJ Software.

Murine catheter implantation model. Prior to implantation, 2-mm sterile catheter pieces (Hospira Sapphire, AP431-01) were pre-colonized for $24 \mathrm{~h}$ at $28^{\circ} \mathrm{C}$ with an overnight culture of $S$. Typhimurium diluted 1:100 in LB No Salt Broth. The catheters were then inserted through percutaneous tunnel incisions into the backs of six- to eight-week-old female BALB/c mice (Jackson Laboratory) using sterile techniques under isoflurane anesthesia. Mice were anesthetized with isoflurane at 24 and $48 \mathrm{~h}$ after catheter insertion and $100 \mu \mathrm{g} 3 \mathrm{H} 3$ was percutaneously injected into the catheter lumens. Some of the mice received $1 \mathrm{mg} / \mathrm{ml}$ of ampicillin (Sigma) mixed into water given ad libitum, starting $24 \mathrm{~h}$ prior to catheter insertion. Mice were euthanized $72 \mathrm{~h}$ after catheter implantation. Excised catheters were stained with Syto9 and Congo Red and visualized as described previously ${ }^{67}$.

A $\beta$ monomers, ADDLs, oligomers, and fibrils. Synthetic $A \beta 42$ monomers biotin-labeled at either the $\mathrm{N}$ or $\mathrm{C}$ terminus were obtained from Anaspec (Fremont, CA). A $\beta$ globular oligomers were prepared as previously described ${ }^{68}$. Briefly, $A \beta 42$ monomer (Life Technologies, Grand Island, NY) was dissolved at $5 \mathrm{mM}$ in dry DMSO, sonicated in a bath sonicator for $30 \mathrm{~min}$, adjusted to $400 \mu \mathrm{M}$ with PBS, following the addition of $1 / 10$ th volume of $2 \%$ SDS, the mixture was incubated at $37^{\circ} \mathrm{C}$ for $6 \mathrm{~h}$. After dilution with three volumes of water, the mixture was incubated for an additional $18 \mathrm{~h}$ at $37^{\circ} \mathrm{C}$ and centrifuged at $3000 \times g$ for $20 \mathrm{~min}$ at RT to remove any $\mathrm{A} \beta 1-42$ fibrils. The supernatant was then concentrated using a Vivaspin centrifugal concentrator with a $30 \mathrm{kDa}$ molecular weight cut-off (Sartorius Stedim, Bohemia, NY), dialyed against PBS, characterized by SDS:PAGE, and stored at $4{ }^{\circ} \mathrm{C}$ for up to 2 weeks. To create stable SPR sensors, oligomers were cross-linked by treatment with $1 \mathrm{mM}$ glutaraldehyde for $15 \mathrm{~min}$ at RT followed by adjusting to $5 \mathrm{mM}$ ethanolamine. The ethanolamine was removed by ultrafiltration using a $10 \mathrm{kDa}$ cut-ff centrifugal concentrator. $\mathrm{A} \beta$ fibrils were prepared by a modification of the method of O'Nuallain and Wetzel ${ }^{50}$. Hexafluoro-2-propanol (HFIP)-treated $\mathrm{A} \beta$ monomer was dissolved in freshly diluted $2 \mathrm{mM} \mathrm{NaOH}$. After gentle agitation, the solution was centrifuged at $10,000 \times g$ for $60 \mathrm{~min}$ to remove large clumps or fibrils. The supernatant was then adjusted to $1 \times$ PBS, $0.05 \%$ sodium azide, and incubated with agitation at $37^{\circ} \mathrm{C}$ for 14 days. Tau PHF were isolated from brain tissue obtained from $\mathrm{AD}$ patients at autopsy using the method of Greenberg and Davies ${ }^{69}$.

Human monoclonal antibody cloning. Human mAbs reactive with $\mathrm{A} \beta$ ADDLs were obtained from a blood sample of a female in her 70s (Patient ID: AD13_7) with a clinical diagnosis of mild to moderate Alzheimer's disease. The sample was collected under a protocol approved by the Main Line Hospitals Institutional Review Board and was consistent with the principles set out in the WMA Declaration of Helsinki and the NIH Belmont Report. Following receipt of informed consent, blood was collected, and $\mathrm{CD} 27^{+}$mononuclear cells were isolated and fused to the B5-6T fusion partner cell line following the published methods ${ }^{70}$. Following HAT selection, hybridoma supernatants were screened by ELISA for secretion of IgGs that bound to ADDLs A $\beta 1-42$ oligomers and antigen- specific IgGs were detected with the HRP-conjugated anti-human IgG Fc fragmentspecific monoclonal antibody (9040-05, Southern Biotechnology). Stable, antigenspecific mAbs (including 4G1,4A6, and 2C10) were isolated by three rounds of cloning in semi-solid medium. For scale-up, hybridomas were adapted to advanced RPMI with 5\% ultra-low IgG medium (Life Technologies) and incubated for 5 days in a 500-ml roller bottle. Filtered supernatants were purified over protein GSepharose (GE Healthcare Life Sciences). Antibody concentrations were determined using a NanoDrop Spectrophotometer (NanoDrop Technologies, Wilmington, DE). The isotype control human mAb 6A (anti-botulinum toxin antibody) was described previously ${ }^{39}$. IgG heavy chain and light chain subtypes were determined by ELISA, and variable domain DNA sequences were obtained as previously described. Sequences were analyzed with the IMGT website (http:// www.imgt.org) $)^{71}$

Biotinylation of sensor ligands. $A \beta$ globular oligomers (ADDLs) were biotin labeled with NHS-PEO ${ }_{12}$-Biotin (Pierce, Rockford, IL) as specified by the manufacturer. The molar excess of biotinylation reagent to $A \beta$ oligomers was between 20 - to 60 -fold; higher ratios were used when the concentration of oligomer was less than $2 \mathrm{mg} / \mathrm{ml}$. After $2 \mathrm{~h}$ at $4{ }^{\circ} \mathrm{C}$, excess biotin reagent was removed by dialysis against PBS. IAPP fibrils, A $\beta$ fibrils, and tau PHF were biotinylated using this same procedure.

SPR measurements. Biotinylated A $\beta$ monomer, oligomer, fibril, tau PHF, and IAPP preparations were each immobilized on the gold surfaces of SensiQ $\mathrm{CO}_{2} \mathrm{H}$ sensors using a neutravidin-biotinylated target molecule immobilization protocol developed by the manufacturer (SensiQ Technologies, Oklahoma City, OK). Briefly, the sample channel was activated for conventional amine coupling by passing $50 \mu \mathrm{l}$ of EDC/NHS activation solution over the channel at $25 \mu \mathrm{l} / \mathrm{min}$. This was followed by a $100 \mu \mathrm{L}$ injection of $100 \mu \mathrm{g} / \mathrm{ml}$ solution of Neutravidin, in $10 \mathrm{mM}$ acetate buffer $\mathrm{pH} 4.5$, over the activated surface for $20 \mathrm{~min}$ at $5 \mu \mathrm{l} / \mathrm{min}$. This was followed by $100 \mu \mathrm{L}$ Biotin tagged $\mathrm{A} \beta(50 \mu \mathrm{g} / \mathrm{ml})$ injected over the Neutravidincoated surface at $25 \mu \mathrm{l} / \mathrm{min}$. After $1 \mathrm{~h}$ equilibration, the sensor was prepared for binding studies with $10 \mathrm{mM}$ phosphoric acid.

For binding assays, $100 \mu \mathrm{lmAb}$ solution was co-injected into the SPR apparatus at a flow rate of $50 \mu \mathrm{l} / \mathrm{min}$. Five minutes after the injection was completed, the sensor surfaces were regenerated with $100 \mu \mathrm{l}$ of $10 \mathrm{mM}$ phosphoric acid at a flow rate of $50 \mu \mathrm{l} / \mathrm{min}$. The binding curves, disassociation constants $\left(K_{\mathrm{d}}\right)$, maximum response $\left(R_{\max }\right)$, and their respective standard deviations were determined using single component fits using Qdat Data Analysis Software (SensiQ Technologies).

Inhibition of fibril formation. We used dynamic light scattering to measure amyloid fibril formation in solution. $Z_{\text {ave }}$ measurements of $0.25 \mathrm{ml}$ of $\mathrm{A} \beta$ globulomers $(50 \mu \mathrm{g} / \mathrm{ml})$ in PBS pH 7.4 were made without or with the addition of $2 \mu \mathrm{g}$ human $\mathrm{mAb}$. The $Z_{\text {ave }}$ measurements were taken for an hour using a Zetasizer Dynamic Light Scattering apparatus (Malvern Panalytical, Malvern UK).

Thioflavin T fibrillization assay. Synthetic peptides that correspond to the fourth and fifth repeats of $\operatorname{CsgA}, \operatorname{Csg} A_{R 4-5}$, and $\operatorname{Csg} A_{R 5-4 N 122 A}$ described previously ${ }^{42}$ were synthesized by Biosynthesis Inc. Polymerization of the synthetic peptides was previously described ${ }^{43}$. To monitor the fibrillization of the synthetic CsgA peptide, $100 \mu \mathrm{M} \operatorname{Csg} \mathrm{A}_{\mathrm{R} 4-5}$, or $100 \mu \mathrm{M} \operatorname{Csg} \mathrm{A}_{\mathrm{R} 5-4 \mathrm{~N} 122 \mathrm{~A}}$ was mixed with an equal volume $10 \mu \mathrm{M}$ ThT in the presence or absence of $0.5 \mathrm{mg} / \mathrm{ml} \mathrm{mAb} 3 \mathrm{H} 3$ or control antibody $6 \mathrm{~A}$ in a black Nunc 96-well plate (Fisher). The plate was sealed with an optical plate cover (Eppendorf). Fluorescence of ThT (excitation $440 \mathrm{~nm} / \mathrm{emission} 490 \mathrm{~nm}$ ) was monitored at $37^{\circ} \mathrm{C}$ using a BMG Labtech POLARstar Omega plater reader. Readings were taken at 8-min intervals for $36 \mathrm{~h}$. Lag time $\left(t_{0}\right)$ was calculated using the following formula: $t_{0}=t_{1 / 2}-2 t_{\mathrm{e}}$, where $t_{1 / 2}$ is the time required to reach half the maximum fluorescence intensity and $t_{\mathrm{e}}$ is the elongation time ${ }^{43}$.

Ethics statement. All animal experiments were performed in AALAC-accredited animal care and use program at the Lewis Katz School of Medicine, with protocol (\#4711) approved by the Temple University Institutional Animal Care and Use Committee in accordance with guidelines set forth by the USDA and PHS Policy on Humane Care and Use of Laboratory Animals under the guidance of the Office of Laboratory Animal Welfare (OLAW).

Statistical analysis. Data were analyzed using GraphPad Prism software. Twotailed Student's $t$-tests were used as appropriate. The $p$ values $<0.05$ were considered significant.

Reporting summary. Further information on research design is available in the Nature Research Reporting Summary linked to this article.

\section{Data availability}

Source data for figures are provided as a Source Data file. Other data generated during and/or analyzed during the current study are available from the corresponding author on request. 
Received: 27 June 2019; Accepted: 17 January 2020;

Published online: 21 February 2020

\section{References}

1. Costerton, J. W., Stewart, P. S. \& Greenberg, E. P. Bacterial biofilms: a common cause of persistent infections. Science 284, 1318-1322 (1999).

2. Donlan, R. M. Biofilms on central venous catheters: is eradication possible? Curr. Top. Microbiol. Immunol. 322, 133-161 (2008).

3. Donlan, R. M. \& Costerton, J. W. Biofilms: survival mechanisms of clinically relevant microorganisms. Clin. Microbiol. Rev. 15, 167-193 (2002).

4. Fatkenheuer, G., Cornely, O. \& Seifert, H. Clinical management of catheterrelated infections. Clin. Microbiol. Infect. 8, 545-550 (2002).

5. Gominet, M., Compain, F., Beloin, C. \& Lebeaux, D. Central venous catheters and biofilms: where do we stand in 2017? APMIS 125, 365-375 (2017).

6. Koo, H., Allan, R. N., Howlin, R. P., Stoodley, P. \& Hall-Stoodley, L. Targeting microbial biofilms: current and prospective therapeutic strategies. Nat. Rev. Microbiol. 15, 740-755 (2017).

7. Letica-Kriegel, A. S. et al. Identifying the risk factors for catheter-associated urinary tract infections: a large cross-sectional study of six hospitals. BMJ Open 9, e022137 (2019).

8. Feasey, N. A. et al. Typhoid fever and invasive nontyphoid salmonellosis, Malawi and South Africa. Emerg. Infect. Dis. 16, 1448-1451 (2010).

9. Goldstein, E. et al. Antimicrobial resistance prevalence, rates of hospitalization with septicemia and rates of mortality with sepsis in adults in different US states. Int. J. Antimicrob. Agents 54, 23-34 (2019).

10. Gordon, M. A. et al. Epidemics of invasive Salmonella enterica serovar enteritidis and S. enterica Serovar Typhimurium infection associated with multidrug resistance among adults and children in Malawi. Clin. Infect. Dis. 46, 963-969 (2008).

11. Crawford, R. W. et al. Gallstones play a significant role in Salmonella spp. gallbladder colonization and carriage. Proc. Natl. Acad. Sci. USA 107, 4353-4358 (2010).

12. Lake, J. G. et al. Pathogen distribution and antimicrobial resistance among pediatric healthcare-associated infections reported to the National Healthcare Safety Network, 2011-2014. Infect. Control Hosp. Epidemiol. 39, 1-11 (2018).

13. See, I., Freifeld, A. G. \& Magill, S. S. Causative organisms and associated antimicrobial resistance in healthcare-associated, central line-associated bloodstream infections from oncology settings, 2009-2012. Clin. Infect. Dis. 62, 1203-1209 (2016).

14. Gustinetti, G. \& Mikulska, M. Bloodstream infections in neutropenic cancer patients: a practical update. Virulence 7, 280-297 (2016).

15. Ejrnaes, K. Bacterial characteristics of importance for recurrent urinary tract infections caused by Escherichia coli. Dan. Med. Bull. 58, B4187 (2011).

16. Zmistowski, B. et al. Prosthetic joint infection caused by gram-negative organisms. J. Arthroplast. 26, 104-108 (2011).

17. Hall, C. W. \& Mah, T. F. Molecular mechanisms of biofilm-based antibiotic resistance and tolerance in pathogenic bacteria. FEMS Microbiol. Rev. 41, 276-301 (2017).

18. Crabbe, A., Jensen, P. O., Bjarnsholt, T. \& Coenye, T. Antimicrobial tolerance and metabolic adaptations in microbial biofilms. Trends Microbiol. 27, 850-863 (2019).

19. Van Acker, H., Van Dijck, P. \& Coenye, T. Molecular mechanisms of antimicrobial tolerance and resistance in bacterial and fungal biofilms. Trends Microbiol. 22, 326-333 (2014).

20. Hathroubi, S., Mekni, M. A., Domenico, P., Nguyen, D. \& Jacques, M. Biofilms: microbial shelters against antibiotics. Microb. Drug Resist. 23, 147-156 (2017).

21. Biesecker, S. G., Nicastro, L. K., Wilson, R. P. \& Tukel, C. The functional amyloid curli protects Escherichia coli against complement-mediated bactericidal activity. Biomolecules 8, 5 (2018)

22. Thurlow, L. R. et al. Staphylococcus aureus biofilms prevent macrophage phagocytosis and attenuate inflammation in vivo. J. Immunol. 186, 6585-6596 (2011).

23. Wolfmeier, H., Pletzer, D., Mansour, S. C. \& Hancock, R. E. W. New perspectives in biofilm eradication. ACS Infect. Dis. 4, 93-106 (2018).

24. Lewis, K. Persister cells. Annu Rev. Microbiol. 64, 357-372 (2010).

25. Fleming, D. \& Rumbaugh, K. The consequences of biofilm dispersal on the host. Sci. Rep. 8, 10738 (2018).

26. Fleming, D. \& Rumbaugh, K. P. Approaches to dispersing medical biofilms. Microorganisms 5, E15 (2017).

27. Barnhart, M. M. \& Chapman, M. R. Curli biogenesis and function. Annu. Rev. Microbiol. 60, 131-147 (2006)

28. Hufnagel, D. A., Tukel, C. \& Chapman, M. R. Disease to dirt: the biology of microbial amyloids. PLoS Pathog. 9, e1003740 (2013).
29. Zogaj, X., Bokranz, W., Nimtz, M. \& Romling, U. Production of cellulose and curli fimbriae by members of the family Enterobacteriaceae isolated from the human gastrointestinal tract. Infect. Immun. 71, 4151-4158 (2003).

30. Chapman, M. R. et al. Role of Escherichia coli curli operons in directing amyloid fiber formation. Science 295, 851-855 (2002).

31. Hammer, N. D., Schmidt, J. C. \& Chapman, M. R. The curli nucleator protein, CsgB, contains an amyloidogenic domain that directs CsgA polymerization. Proc. Natl. Acad. Sci. USA 104, 12494-12499 (2007).

32. Wang, X., Smith, D. R., Jones, J. W. \& Chapman, M. R. In vitro polymerization of a functional Escherichia coli amyloid protein. J. Biol. Chem. 282, 3713-3719 (2007).

33. Zhou, Y., Smith, D. R., Hufnagel, D. A. \& Chapman, M. R. Experimental manipulation of the microbial functional amyloid called curli. Methods Mol. Biol. 966, 53-75 (2013).

34. Nicastro, L. K. et al. Cytotoxic curli intermediates form during Salmonella biofilm development. J. Bacteriol. 201, https://doi.org/10.1128/JB.00095-19 (2019).

35. Tursi, S. A. \& Tukel, C. Curli-containing enteric biofilms inside and out: matrix composition, immune recognition, and disease implications. Microbiol. Mol. Biol. Rev. 82, e00028-18 (2018).

36. Wang, X., Hammer, N. D. \& Chapman, M. R. The molecular basis of functional bacterial amyloid polymerization and nucleation. J. Biol. Chem. 283, 21530-21539 (2008).

37. Knowles, T. P., Vendruscolo, M. \& Dobson, C. M. The amyloid state and its association with protein misfolding diseases. Nat. Rev. Mol. Cell Biol. 15, 384-396 (2014)

38. Levites, Y. et al. A human monoclonal IgG that binds abeta assemblies and diverse amyloids exhibits anti-amyloid activities in vitro and in vivo. $J$. Neurosci. 35, 6265-6276 (2015).

39. Adekar, S. P. et al. Hybridoma populations enriched for affinity-matured human IgGs yield high-affinity antibodies specific for botulinum neurotoxins. J. Immunol. Methods 333, 156-166 (2008).

40. Nishimori, J. H. et al. Microbial amyloids induce interleukin 17A (IL-17A) and IL-22 responses via Toll-like receptor 2 activation in the intestinal mucosa. Infect. Immun. 80, 4398-4408 (2012).

41. Xue, C., Lin, T. Y., Chang, D. \& Guo, Z. Thioflavin T as an amyloid dye: fibril quantification, optimal concentration and effect on aggregation. R. Soc. Open Sci. 4, 160696 (2017)

42. Tukel, C. et al. Responses to amyloids of microbial and host origin are mediated through Toll-like receptor 2. Cell Host Microbe 6, 45-53 (2009).

43. Gallo, P. M. et al. Amyloid-DNA composites of bacterial biofilms stimulate autoimmunity. Immunity 42, 1171-1184 (2015)

44. Steinberg, J. P., Robichaux, C., Tejedor, S. C., Reyes, M. D. \& Jacob, J. T. Distribution of pathogens in central line-associated bloodstream infections among patients with and without neutropenia following chemotherapy: evidence for a proposed modification to the current surveillance definition. Infect. Control Hosp. Epidemiol. 34, 171-175 (2013).

45. Hung, C. et al. Escherichia coli biofilms have an organized and complex extracellular matrix structure. MBio 4, e00645-00613 (2014).

46. Romero, D., Aguilar, C., Losick, R. \& Kolter, R. Amyloid fibers provide structural integrity to Bacillus subtilis biofilms. Proc. Natl Acad. Sci. USA 107 2230-2234 (2010)

47. Schwartz, K., Syed, A. K., Stephenson, R. E., Rickard, A. H. \& Boles, B. R. Functional amyloids composed of phenol soluble modulins stabilize Staphylococcus aureus biofilms. PLoS Pathog. 8, e1002744 (2012).

48. Cegelski, L. et al. Small-molecule inhibitors target Escherichia coli amyloid biogenesis and biofilm formation. Nat. Chem. Biol. 5, 913-919 (2009).

49. Kayed, R. et al. Common structure of soluble amyloid oligomers implies common mechanism of pathogenesis. Science 300, 486-489 (2003).

50. O’Nuallain, B. \& Wetzel, R. Conformational Abs recognizing a generic amyloid fibril epitope. Proc. Natl Acad. Sci. USA 99, 1485-1490 (2002).

51. Krishnan, R. et al. Conserved features of intermediates in amyloid assembly determine their benign or toxic states. Proc. Natl. Acad. Sci. USA 109, 11172-11177 (2012)

52. Zhao, M. et al. Pan-amyloid oligomer specific scFv antibody attenuates memory deficits and brain amyloid burden in mice with Alzheimer's disease. Curr. Alzheimer Res. 11, 69-78 (2014)

53. Perov, S. et al. Structural insights into curli CsgA cross-beta fibril architecture inspire repurposing of anti-amyloid compounds as anti-biofilm agents. PLoS Pathog. 15, e1007978 (2019).

54. Jain, N. et al. Inhibition of curli assembly and Escherichia coli biofilm formation by the human systemic amyloid precursor transthyretin. Proc. Natl. Acad. Sci. USA 114, 12184-12189 (2017).

55. DeBenedictis, E. P., Liu, J. \& Keten, S. Adhesion mechanisms of curli subunit CsgA to abiotic surfaces. Sci. Adv. 2, el600998 (2016).

56. Ryu, J. H., Kim, H., Frank, J. F. \& Beuchat, L. R. Attachment and biofilm formation on stainless steel by Escherichia coli O157:H7 as affected by curli production. Lett. Appl. Microbiol. 39, 359-362 (2004). 
57. Demattos, R. B. et al. A plaque-specific antibody clears existing beta-amyloid plaques in Alzheimer's disease mice. Neuron 76, 908-920 (2012).

58. Hrncic, R. et al. Antibody-mediated resolution of light chain-associated amyloid deposits. Am. J. Pathol. 157, 1239-1246 (2000).

59. Pepys, M. B. et al. Targeted pharmacological depletion of serum amyloid P component for treatment of human amyloidosis. Nature 417, 254-259 (2002).

60. Dueholm, M. S., Albertsen, M., Otzen, D. \& Nielsen, P. H. Curli functional amyloid systems are phylogenetically widespread and display large diversity in operon and protein structure. PLoS ONE 7, e51274 (2012).

61. Stojiljkovic, I., Baumler, A. J. \& Heffron, F. Ethanolamine utilization in Salmonella Typhimurium: nucleotide sequence, protein expression, and mutational analysis of the cchA cchB eutE eutJ eutG eutH gene cluster. $J$. Bacteriol. 177, 1357-1366 (1995).

62. Raffatellu, M. et al. The Vi capsular antigen of Salmonella enterica serotype Typhi reduces Toll-like receptor-dependent interleukin-8 expression in the intestinal mucosa. Infect. Immun. 73, 3367-3374 (2005).

63. Humphries, A. D. et al. The use of flow cytometry to detect expression of subunits encoded by 11 Salmonella enterica serotype Typhimurium fimbrial operons. Mol. Microbiol. 48, 1357-1376 (2003).

64. Crawford, R. W., Gibson, D. L., Kay, W. W. \& Gunn, J. S. Identification of a bile-induced exopolysaccharide required for Salmonella biofilm formation on gallstone surfaces. Infect. Immun. 76, 5341-5349 (2008).

65. Tukel, C. et al. CsgA is a pathogen-associated molecular pattern of Salmonella enterica serotype Typhimurium that is recognized by Toll-like receptor 2. Mol. Microbiol. 58, 289-304 (2005).

66. Oppong, G. O. et al. Biofilm-associated bacterial amyloids dampen inflammation in the gut: oral treatment with curli fibres reduces the severity of hapten-induced colitis in mice. NPJ Biofilms Microbiomes 1, 15019 (2015).

67. Tursi, S. A. et al. Bacterial amyloid curli acts as a carrier for DNA to elicit an autoimmune response via TLR2 and TLR9. PLoS Pathog. 13, e1006315 (2017).

68. Barghorn, S. et al. Globular amyloid beta-peptide oligomer-a homogenous and stable neuropathological protein in Alzheimer's disease. J. Neurochem. 95, 834-847 (2005).

69. Greenberg, S. G. \& Davies, P. A preparation of Alzheimer paired helical filaments that displays distinct tau proteins by polyacrylamide gel electrophoresis. Proc. Natl Acad. Sci. USA 87, 5827-5831 (1990).

70. Puligedda, R. D. et al. Characterization of human monoclonal antibodies that neutralize multiple poliovirus serotypes. Vaccine 35, 5455-5462 (2017).

71. Lefranc, M. P. et al. IMGT(R), the International ImMunoGeneTics information system(R) 25 years on. Nucleic Acids Res. 43, D413-D422 (2015).

\section{Acknowledgements}

We thank Chandana Devi for technical support. Work in the CT lab was supported by the National Institutes of Health, National Institute of Allergy and Infectious Diseases Grants AI137541, AI125429, AI126133, AI132996 and partly by Immunome (supplementary studies Fig. S3). Work in the SD lab was supported by National Institutes of Health, National Institute of Allergy and Infectious Diseases R21 AI119368-01A1, and the Lankenau Institute for Medical Research. S.D. holds the Joseph and Ray Gordon

Chair in Clinical Oncology Research, which was established by the Gordon family and the Lankenau Medical Center. Work in the NR laboratory was supported in part by the National Institutes of Health, National Institute on Aging 5U01AG010483-20.

\section{Author contributions}

S.A.T. and R.D.P.: conceptualization, methodology, investigation, and writing. P.S. conceptualization and investigation. L.K.N., A.L.M., and C.Q.: investigation and methodology. S.G.: supervision. N.R.: supervision and funding acquisition. B.A.B.: conceptualization, methodology, supervision, and writing-original draft preparation. S.K.D and C.T.: conceptualization, methodology, funding acquisition, project administration, supervision, writing-original draft preparation, review, and editing.

\section{Competing Interests}

The human mAb $3 \mathrm{H} 3$ has been licensed to Immunome, Inc., and SD is on the Immunome Scientific Advisory Board and holds Immunome Stock. S.D. is an inventor of the $3 \mathrm{H} 3 \mathrm{mAb}$ and therefore may be eligible to receive royalty payments. C.T. and S.D. are inventors on a PCT patent application based on this work. The remaining authors do not declare any competing interests.

\section{Additional information}

Supplementary information is available for this paper at https://doi.org/10.1038/s41467020-14685-3.

Correspondence and requests for materials should be addressed to S.K.D. or Ç.T.

Peer review information Nature Communications thanks Tom Coenye and the other, anonymous, reviewer(s) for their contribution to the peer review of this work. Peer reviewer reports are available.

Reprints and permission information is available at http://www.nature.com/reprints

Publisher's note Springer Nature remains neutral with regard to jurisdictional claims in published maps and institutional affiliations.

Open Access This article is licensed under a Creative Commons Attribution 4.0 International License, which permits use, sharing, adaptation, distribution and reproduction in any medium or format, as long as you give appropriate credit to the original author(s) and the source, provide a link to the Creative Commons license, and indicate if changes were made. The images or other third party material in this article are included in the article's Creative Commons license, unless indicated otherwise in a credit line to the material. If material is not included in the article's Creative Commons license and your intended use is not permitted by statutory regulation or exceeds the permitted use, you will need to obtain permission directly from the copyright holder. To view a copy of this license, visit http://creativecommons.org/ licenses/by/4.0/

(C) The Author(s) 2020 